\title{
EULER-LAGRANGE CHANGE OF VARIABLES IN CONSERVATION LAWS
}

\author{
YUE-JUN PENG \\ Laboratoire de Mathématiques, CNRS UMR 6620 \\ Université Blaise Pascal (Clermont-Ferrand 2), 63177 Aubière cedex, France \\ peng@math.univ-bpclermont.fr
}

\begin{abstract}
We introduce a new method for studying the Cauchy problem for systems of conservation laws in one space dimension. This method is based on the equivalence of the Cauchy problems in Eulerian and Lagrangian coordinates, as regards the existence and uniqueness of entropy solutions. The main idea is to solve the problem in Lagrangian coordinates and determine the transformation linking the two coordinates. The main contributions are the uniqueness and explicit entropy solutions. Applications include the Keyfitz-Kranzer system, the Born-Infeld equations and linear Lagrangian systems which are linear in Lagrangian coordinates. For these examples, the existence and uniqueness of solutions in $L^{\infty}$ are obtained in explicit expressions. The linear Lagrangian system contains examples such as the equations of pressureless gas dynamics, all $2 \times 2$ linearly degenerate systems and the augmented Born-Infeld equations. In particular, we deduce the existence and uniqueness of entropy solutions of the Cauchy problem for the Born-Infeld equations. An explicit formula of its entropy solution is also provided.
\end{abstract}

Keywords. Conservation laws, change of variables, explicit entropy solutions, uniqueness

AMS Subject Classification (2000): 35L65, 76N15, 35Q35

\section{INTRODUCTION}

Both Eulerian and Lagrangian coordinates are used for the description of the evolution of one-dimensional flow of a compressible and inviscid gas. The equations of gas dynamics have a simple form in Lagrangian coordinates and are often treated in those in mathematical analysis instead of in Eulerian coordinates. See for instance [34, 35, 30]. These two coordinate systems are linked by a nonlinear transformation depending on unknown variables $[15,24]$. The transformation is abbreviated by E-L. It possesses many interesting properties (see $[42,44]$ ).

For a class of systems of conservation laws, after an E-L transformation we obtain a system in Lagrangian coordinates. It is easy to see that smooth solutions of the systems in the two coordinate systems are equivalent. In 1987, Wagner gave a rigorous justification of the equivalence of entropy solutions in $L^{\infty}$ of the systems in the two coordinate systems [44]. His result shows that the choice of coordinates is not important and one may work 
with either of them. However, in his work the initial data have not been taken into account and the equivalence of the Cauchy problem in the two systems has not been investigated.

In this paper, we prove that the Cauchy problems in the two coordinate systems are still equivalent as regards the existence and uniqueness of entropy solutions in $L^{\infty}$. An explicit relation between the entropy solutions in the two coordinate systems is exhibited. These results are given in Theorem 2.1 in the general case. They reveal that when the transformation is known, the entropy solution in Eulerian coordinates is obtained explicitly from the entropy solution in Lagrangian coordinates and vice versa. Note that the E-L transformation is uniquely determined by the entropy solution and its initial data. Thus, if the system in Lagrangian coordinates has a simple form, it is possible to solve the Cauchy problem for the original system by determining the transformation.

The E-L transformation is induced by one of the conservation equations. For the linearly degenerate system, entropy inequalities for all convex entropies become entropy equalities for all entropies. This provides many choices of conservation laws for making transformations. This idea is used throughout the applications in Sections 3-6.

The above considerations have some consequences in applications. The first application concerns the Keyfitz-Kranzer system of two equations for which one characteristic field is genuinely nonlinear and the other is linearly degenerate [28]. In a very simple way we establish the existence and uniqueness of entropy solutions for the Cauchy problem. For such a system with convex flux functions, an explicit formula of the entropy solution is provided with the help of the Lax formula for scalar conservation law.

The second application concerns linear Lagrangian systems, a class of systems which are linear in Lagrangian coordinates (see Definition 3.1). For such a hyperbolic system, we give a precise description of its mathematical structure on the eigenvalues, linear degeneracy of the characteristic fields, classical Riemann invariants and entropy-entropy flux pairs. Strictly convex entropies are also constructed. From the explicit entropy solution of the Cauchy problem for the linear system in Lagrangian coordinates, we deduce a unique E-L transformation which yields a unique entropy solution of its Cauchy problem with an explicit expression. This expression is of the form : $w_{i}(t, x)=w_{i}^{0}\left(\alpha_{i}(t, x)\right)$, where $w_{i}$ is an $i$-th classical Riemann invariant of the system, $w_{i}^{0}$ is its initial value and $\alpha_{i}$ is determined explicitly by the system and the initial data. Therefore, a maximum principle can be applied to each $w_{i}$. Furthermore, we show that the entropy solution is weakly stable in $L^{\infty}\left(\mathbb{R}^{+} \times \mathbb{R}\right)$ and satisfies the entropy equalities for all entropy-entropy flux pairs of the system.

We present three physical examples of linear Lagrangian systems. They are the system of pressureless gas dynamics, all $2 \times 2$ linearly degenerate systems and the augmented Born-Infeld (abbreviated to ABI) equations introduced by Brenier [9]. These systems are not necessarily strictly hyperbolic. For the system of pressureless gas dynamics which is not even hyperbolic, the local existence of solutions in $L^{\infty}$ with explicit expressions is shown under a partial regularity assumption on the initial velocity. The solution is global if the initial velocity is a non-decreasing function. The $2 \times 2$ linearly degenerate system and the ABI equations are two hyperbolic linear Lagrangian systems. Thus our results can be immediately applied to them. The main application is the existence and uniqueness of entropy solutions of the Cauchy problem for the Born-Infeld (abbreviated by BI) system. The later is not a linear Lagrangian system. The results for this system are obtained from the entropy solution and a further study on the entropy-entropy flux 
pairs of the ABI equations. An explicit formula of the entropy solution of the BI equations is also given.

Finally, we remark that the equivalence of the two coordinate systems in several space dimensions is discussed in $[16,17,45]$. See also [20] for a numerical investigation in two dimensional equations of gas dynamics and the references therein. However, due to the essential difference between the systems of conservation laws in one dimension and several dimensions, it is not so obvious to obtain the results of this paper in several dimensions. A further study on the problem will be given in a forthcoming work.

This paper is organized as follows. In the next section, we consider the change of variables and establish the equivalence of entropy solutions of the Cauchy problems in the two coordinate systems. The main result of this section is Theorem 2.1. In Section 3 we apply Theorem 2.1 to the Keyfitz-Kranzer system. Section 4 is devoted to the study of the linear Lagrangian system with its first example: pressureless gas dynamics. Sections 5 and 6 contain another two examples of linear Lagrangian systems : the $2 \times 2$ linearly degenerate system and the ABI equations. At this stage the BI equations are studied. In

the Appendix, we give the proof of Lemma 6.2 which is a key step to obtain the main results for the $\mathrm{BI}$ equations.

\section{Equivalence of ENTROPy SOlutions in TWO COORDinATE Systems}

2.1. Definitions. Consider the Cauchy problem for a system of conservation laws :

$$
\partial_{t} u+\partial_{x} f(u)=0, \quad t>0, x \in \mathbb{R},
$$

subject to an initial condition :

$$
t=0: \quad u=u^{0}(x), \quad x \in \mathbb{R} .
$$

Here $u=\left(u_{1}, u_{2}, \cdots, u_{n}\right)^{t}, u^{0}=\left(u_{1}^{0}, u_{2}^{0}, \cdots, u_{n}^{0}\right)^{t} \in L^{\infty}(\mathbb{R})$, and $f=\left(f_{1}, f_{2}, \cdots, f_{n}\right)^{t}$ is a smooth function from a domain $U \subset \mathbb{R}^{n}$ to $\mathbb{R}^{n}$. We denote by $\lambda_{1}(u), \lambda_{2}(u), \cdots, \lambda_{n}(u)$ the eigenvalues of the system (2.1), i.e., the eigenvalues of the matrix $A(u)=f^{\prime}(u)$ and by $r_{1}(u), r_{2}(u), \cdots, r_{n}(u)$ the corresponding right eigenvectors. The following notions are fundamental, see [29] for instance. We say that the system (2.1) is hyperbolic in $U$ if $\lambda_{1}(u), \lambda_{2}(u), \cdots, \lambda_{n}(u)$ are real and $A(u)$ is diagonalizable for all $u \in U$. It is strictly hyperbolic if $\lambda_{1}(u), \lambda_{2}(u), \cdots, \lambda_{n}(u)$ are all distinct. The $i$-th characteristic $\lambda_{i}(u)$ is genuinely nonlinear in the sense of P.D.Lax if $\nabla \lambda_{i}(u) \cdot r_{i}(u) \neq 0$ and is linearly degenerate if $\nabla \lambda_{i}(u) \cdot r_{i}(u)=0$ for all $u \in U$. If all the characteristics are linearly degenerate, the system (2.1) is said to be linearly degenerate.

It is known that solutions of the system (2.1) develop singularities in a finite time even if the initial data are smooth. As a consequence one has to seek global existence of weak solutions in the sense of distributions. In order to guarantee the uniqueness of weak solutions an entropy condition is imposed. A function $u \in L_{l o c}^{\infty}\left(\mathbb{R}^{+} \times \mathbb{R}\right)$ is called entropy solution if for all entropy-entropy flux pairs $(E, F)$ with $E$ convex it satisfies

$$
\partial_{t} E(u)+\partial_{x} F(u) \leq 0,
$$

in the sense of distributions. 
2.2. Equivalence of systems. Let $u \in L^{\infty}\left(\mathbb{R}^{+} \times \mathbb{R}\right)$ be an entropy solution of the system (2.1) with $u_{1}(t, x) \geq \underline{u}_{1}>0$. The variable $u_{1}$ plays a special role in the study. In physical models it may stand for the density, energy density etc. Using the first equation in (2.1) for $u_{1}$, we may take an E-L transformation from $(t, x)$ to $(s, y)$ :

$$
(s, y)=(t, Y(t, x)) \quad \text { and } \quad d y=u_{1} d x-f_{1}(u) d t .
$$

This change of variables can also be written as :

$$
s=t \quad \text { and } y=\int_{X_{1}(t)}^{x} u_{1}(t, \xi) d \xi \quad \text { with } \quad X_{1}^{\prime}(t)=\frac{f_{1}\left(u\left(t, X_{1}(t)\right)\right)}{u_{1}\left(t, X_{1}(t)\right)} .
$$

It is unique if $Y(0, x)$ or $X_{1}(0)$ is given. Then in Lagrangian coordinates $(s, y)$, the system (2.1) reads (see [44]) :

$$
\left\{\begin{array}{l}
\partial_{s}\left(\frac{1}{\tilde{u}_{1}}\right)-\partial_{y}\left(\frac{f_{1}(\tilde{u})}{\tilde{u}_{1}}\right)=0, \\
\partial_{s}\left(\frac{\tilde{u}_{i}}{\tilde{u}_{1}}\right)+\partial_{y}\left(f_{i}(\tilde{u})-\frac{f_{1}(\tilde{u}) \tilde{u}_{i}}{\tilde{u}_{1}}\right)=0 \quad(2 \leq i \leq n),
\end{array}\right.
$$

for $s>0$ and $y \in \mathbb{R}$. Here and in what follows we denote by $\tilde{u}$ the variable $u$ in Lagrangian coordinates, i.e., $\tilde{u}(s, y)=u(t, x)$. More generally, for an entropy-entropy flux pair $(E, F)$ of the system (2.1), let us define

$$
\tilde{E}(v)=E(u) / u_{1}, \quad \tilde{F}(v)=F(u)-f_{1}(u) E(u) / u_{1},
$$

with

$$
v_{1}=\frac{1}{u_{1}}, \quad v_{i}=\frac{u_{i}}{u_{1}} \quad(2 \leq i \leq n), \quad v=\left(v_{1}, v_{2}, \cdots, v_{n}\right)^{t} .
$$

For $u_{1}>0$ the function $\varphi: u \longmapsto v$ is a $C^{\infty}$-diffeomorphism with the property $\varphi \circ \varphi=I_{d}$. From [44] we know that $(E, F)$ is an entropy-entropy flux pair of $(2.1)$ if and only if $(\tilde{E}, \tilde{F})$ is an entropy-entropy flux pair for (2.5), and $E$ is convex with respect to $u$ if and only if $\tilde{E}$ is convex with respect to $v$. Hence, in Lagrangian coordinates the entropy condition (2.3) is equivalent to

$$
\partial_{s} \tilde{E}(\tilde{v})+\partial_{y} \tilde{F}(\tilde{v}) \leq 0, \quad \tilde{E} \text { convex. }
$$

For linearly degenerate systems the Rankine-Hugoniot conditions are independent of the choice of the conservation laws among all entropy-entropy flux pairs. Therefore, it is expected that the entropy solution satisfies the entropy equality

$$
\partial_{t} E(u)+\partial_{x} F(u)=0
$$

for all entropy-entropy flux pairs $(E, F)$. This condition is equivalent to

$$
\partial_{s} \tilde{E}(\tilde{v})+\partial_{y} \tilde{F}(\tilde{v})=0 .
$$

Definition 2.1. A function $u \in L_{\text {loc }}^{\infty}\left(\mathbb{R}^{+} \times \mathbb{R}\right)$ is called an entropy solution of the linear degenerate system (2.1) if (2.9) holds for all entropy-entropy flux pairs (E, F).

As we will see in Sections 4-6, condition (2.9) is satisfied for $L^{\infty}$ weak solutions of the linear Lagrangian system and the BI equations. When only a part of the characteristic fields is linearly degenerate, we require the entropy equality (2.9) for all entropy-entropy flux pairs corresponding to the linearly degenerate fields. This is the case of the KeyfitzKranzer system (see section 3). 
The E-L transformation possesses many remarkable properties. In particular, if we denote by $\tilde{\lambda}_{i}(\tilde{u})$ the $i$-th eigenvalue of the system (2.5), from the second equation in (2.4), we obtain

which gives,

$$
\frac{d y}{d t}=u_{1} \frac{d x}{d t}-f_{1}(u)
$$

$$
\tilde{\lambda}_{i}(u)=u_{1} \lambda_{i}(u)-f_{1}(u) .
$$

Moreover, let $A(u)=f^{\prime}(u)$. The non-conservative equations $\partial_{t} u+A(u) \partial_{x} u=0$ are equivalent to $\partial_{s} \tilde{u}+\bar{A}(\tilde{u}) \partial_{y} \tilde{u}=0$ with $\bar{A}(u)=u_{1} A(u)-f_{1}(u) I_{n}$. Therefore, $A(u)$ and $\bar{A}(u)$ have the same eigenvectors.

In the following proposition are listed the main equivalence properties between the systems (2.1) and (2.5). The proof can be found in [42,44].

Proposition 2.1. The E-L transformation preserves the (strict) hyperbolicity, convexity of entropies with relation (2.6), entropy solutions, and for each $i=1,2, \cdots, n$, the existence of an $i$-th classical Riemann invariant and genuine nonlinearity or linear degeneracy of the $i$-th characteristic field of systems. Finally, the relation of the eigenvalues of the systems (2.1) and (2.5) in the two coordinate systems is given by (2.11).

Remark 2.1. If $u_{1}$ is a function of $\left(u_{2}, \cdots, u_{n}\right)$ and the first equation $\partial_{t} u_{1}+\partial_{x} f_{1}(u)=0$ in (2.1) is exact and is derived from the sub-system composed of the $n-1$ equations in (2.1) with variables $\left(u_{2}, \cdots, u_{n}\right)$, then this sub-system is equivalent to the sub-system without the first equation in (2.5), in the sense of Proposition 2.1.

2.3. Equivalence of the Cauchy problems. From the definition (2.4) and $\tilde{u}(s, y)=$ $u(t, x)$, we have $\tilde{u}(t, Y(t, x))=u(t, x)$. If we set

$$
Y(0, x)=Y_{0}(x) \stackrel{\text { def }}{=} \int_{0}^{x} u_{1}^{0}(\xi) d \xi
$$

then for each given $u \in L^{\infty}\left(\mathbb{R}^{+} \times \mathbb{R}\right),(2.4)$ and (2.12) define a unique E-L transformation. Note that the choice of $Y_{0}$ by (2.12) is compatible with (2.4). Obviously, for $u_{1}^{0}(x) \geq \underline{u}_{1}>$ $0, Y_{0}$ is strictly increasing, Lipschitzian and bijective from $\mathbb{R}$ to $\mathbb{R}$. So is its inverse function $X_{0}$. Similarly, for all $t>0, x \longmapsto Y(t, x)$ is bijective from $\mathbb{R}$ to $\mathbb{R}$ and $(t, x) \longmapsto Y(t, x)$ is Lipschitzian. Concerning the inverse function of $Y(t, \cdot)$, we have the following result.

Lemma 2.1. Assume $u^{0} \in L^{\infty}(\mathbb{R})$ with $u_{1}^{0}(x) \geq \underline{u}_{1}>0$, a.e. $x \in \mathbb{R}$. Let $u \in L^{\infty}\left(\mathbb{R}^{+} \times \mathbb{R}\right)$ be an entropy solution of (2.1)-(2.2) with $u_{1}(t, x) \geq \underline{u}_{1}>0$, a.e. $(t, x) \in \mathbb{R}^{+} \times \mathbb{R}$. Let $\tilde{u}(t, y)=u(t, x)$ and $x=X(t, y)$ be the unique solution of

$$
d x=\frac{1}{\tilde{u}_{1}} d y+\frac{f_{1}(\tilde{u})}{\tilde{u}_{1}} d t, \quad X(0, y)=X_{0}(y) .
$$

Then $X(t, \cdot)=Y^{-1}(t, \cdot)$ for all $t \geq 0$.

Proof. Let us just compute, for all $t>0$,

$$
\begin{aligned}
\frac{d Y(t, X(t, y))}{d t} & =\frac{\partial Y(t, X(t, y))}{\partial t}+\frac{\partial Y(t, X(t, y))}{\partial x} \cdot \frac{\partial X(t, y)}{\partial t} \\
& =\left(-f_{1}(\tilde{u})+\tilde{u}_{1} \cdot \frac{f_{1}(\tilde{u})}{\tilde{u}_{1}}\right)(t, X(t, y))=0 .
\end{aligned}
$$


Since $Y(0, X(0, y))=Y_{0}\left(X_{0}(y)\right)=y$, we obtain $Y(t, X(t, y))=y$ for all $t \geq 0$. Similarly, $X(t, Y(t, x))=x$. Hence, $X(t, \cdot)=Y^{-1}(t, \cdot)$ for all $t \geq 0$.

Taking $t=0$ in $\tilde{u}(t, y)=u(t, X(t, y))$ gives $\tilde{u}(0, y)=u^{0}\left(X_{0}(y)\right)$. Thus by Wagner's result [44] which uses the E-L transformation (2.4) in weak formulations of the systems, we obtain the following equivalence result for the Cauchy problems.

Theorem 2.1. Let the assumptions of Lemma 2.1 hold and $(t, x) \longmapsto(s, y)$ be the unique $E$ - $L$ transformation defined by (2.4) and (2.12). Then $\tilde{u}(t, y)=u(t, X(t, y))$ is an entropy solution of (2.5) satisfying the initial condition $\left.\tilde{u}\right|_{s=0}=u^{0}\left(X_{0}\right)$, where $X_{0}$ and $X(t, \cdot)$ are the inverse functions of $Y_{0}$ and $Y(t, \cdot)$, respectively.

Furthermore, if the entropy solution of the Cauchy problem for the system (2.5) is unique, then the entropy solution $u \in L^{\infty}\left(\mathbb{R}^{+} \times \mathbb{R}\right)$ of (2.1)-(2.2) with $u_{1}(t, x) \geq \underline{u}_{1}>0$, a.e. $(t, x) \in \mathbb{R}^{+} \times \mathbb{R}$ is unique.

Proof. The proof of the first part of Theorem 2.1 is clear. It suffices to give the proof of its second part.

Let $u \in L^{\infty}\left(\mathbb{R}^{+} \times \mathbb{R}\right)$ be an entropy solution of (2.1)-(2.2) with $u_{1}(t, x) \geq \underline{u}_{1}>0$, a.e. $(t, x) \in \mathbb{R}^{+} \times \mathbb{R}$ and $(t, x) \longmapsto\left(t, Y_{u}(t, x)\right)$ be the unique E-L transformation with $y=Y_{u}(t, x)$ being the unique Lipschitzian solution of (2.4) and (2.12). From Lemma 2.1, the inverse function $X_{u}(t, \cdot)$ of $Y_{u}(t, \cdot)$ is given by (2.13). Then the first part of Theorem 2.1 shows that $\tilde{u}(t, y)=u\left(t, X_{u}(t, y)\right)$ is an entropy solution of (2.5) with initial value $\tilde{u}^{0}=u^{0}\left(X_{0}\right)$. From the uniqueness assumption of the Cauchy problem to the system (2.5), $\tilde{u}$ depends only on $u^{0}$ and $f$ but is independent of $u$ for $t>0$. It follows from (2.13) that $X_{u}$ is independent of $u$. So is $Y_{u}$ and we may write $Y_{u}=Y$. Thus, any entropy solution $u$ of (2.1)-(2.2) with $u_{1}(t, x) \geq \underline{u}_{1}>0$ has the same expression $u(t, x)=\tilde{u}(t, Y(t, x))$. This proves the uniqueness of entropy solutions to the Cauchy problem (2.1)-(2.2).

By Lemma 2.1, Theorem 2.1 shows the equivalence on the existence and uniqueness of entropy solutions for the Cauchy problems in the two coordinate systems. This result can be immediately applied to the Cauchy problem for the equations of gas dynamics in Eulerian coordinates :

$$
\left\{\begin{array}{l}
\partial_{t} \rho+\partial_{x}(\rho u)=0 \\
\partial_{t}(\rho u)+\partial_{x}\left(\rho u^{2}+p\right)=0 \\
\partial_{t}\left(\rho\left(e+\frac{1}{2} u^{2}\right)\right)+\partial_{x}\left(\left(\rho\left(e+\frac{1}{2} u^{2}\right)+p\right) u\right)=0, \quad t>0, x \in \mathbb{R} \\
t=0:(\rho, u, e)=\left(\rho^{0}, u^{0}, e^{0}\right)(x), \quad x \in \mathbb{R}
\end{array}\right.
$$

and in Lagrangian coordinates :

$$
\left\{\begin{array}{l}
\partial_{s} \tilde{v}-\partial_{y} \tilde{u}=0 \\
\partial_{s} \tilde{u}+\partial_{y} \tilde{p}(\tilde{v}, \tilde{e})=0 \\
\partial_{s}\left(\tilde{e}+\frac{1}{2} \tilde{u}^{2}\right)+\partial_{y}(\tilde{p}(\tilde{v}, \tilde{e}) \tilde{u})=0, \quad s>0, y \in \mathbb{R} \\
s=0:(\tilde{v}, \tilde{u}, \tilde{e})=\left(\tilde{v}^{0}, \tilde{u}^{0}, \tilde{e}^{0}\right)(y), \quad y \in \mathbb{R},
\end{array}\right.
$$

through the change of variables (2.4) and (2.12) with $u_{1}=\rho, f_{1}(\rho, u)=\rho u, \tilde{p}(v, e)=$ $p(1 / v, e)$ and $(\tilde{v}, \tilde{u}, \tilde{e})(s, y)=(1 / \rho, u, e)(t, x)$. In the above equations, $\rho>0, u, e>0$ and 
$p=p(\rho, e)$ are the density, velocity, specific internal energy and pressure, respectively. Thus, $(\rho, u, e) \in L^{\infty}\left(\mathbb{R}^{+} \times \mathbb{R}\right)$ with $\rho \geq \rho>0$ is an entropy solution of (2.14) if and only if $(\tilde{v}, \tilde{u}, \tilde{e})$ is an entropy solution of $\overline{(2.15)}$ for which the initial data are linked by $\left(\tilde{v}^{0}, \tilde{u}^{0}, \tilde{e}^{0}\right)=\left(1 / \rho^{0}, u^{0}, e^{0}\right) \circ X_{0}$ or $\left(\rho^{0}, u^{0}, e^{0}\right)=\left(1 / \tilde{v}^{0}, \tilde{u}^{0}, \tilde{e}^{0}\right) \circ Y_{0}$. The entropy solution of (2.14) is unique if and only if that of (2.15) is unique. In particular, the $L^{\infty}$ entropy solutions of the Cauchy problems for the isentropic gas dynamics equations are equivalent in Eulerian and Lagrangian coordinate systems.

Notice that the above equivalence result is also valid for systems of conservation laws with source terms provided that the source term does not appear in the conservation law for $u_{1}>0$. Hence, Theorem 2.1 can be applied to one-dimensional Navier-Stokes equations and Shallow Water equations. Further applications are given in the next sections.

\section{A Keyfitz-Kranzer MOdeL}

In mathematical modeling of enhanced oil recovery, the Keyfitz-Kranzer system is often used [28]. A simplified version of this system reads :

$$
\partial_{t} u+\partial_{x}(u \phi(r))=0, \quad t>0, x \in \mathbb{R},
$$

subject to an initial condition :

$$
t=0: u=u^{0}(x), \quad x \in \mathbb{R},
$$

where $u=\left(u_{1}, u_{2}\right)^{t}, r=|u|=\sqrt{u_{1}^{2}+u_{2}^{2}}$, and $\phi: \mathbb{R}^{+} \longrightarrow \mathbb{R}$ is a smooth function.

The two eigenvalues of the system are :

$$
\lambda_{1}(u)=(r \phi(r))^{\prime}, \quad \lambda_{2}(u)=\phi(r) .
$$

We check easily that the second characteristic field is linearly degenerate. When $(r \phi(r))^{\prime \prime} \equiv$ 0, i.e., $\phi(r)=C_{1} / r+C_{2}$ for $r>0$, with $C_{1}, C_{2} \in \mathbb{R}$, the first characteristic field is also linearly degenerate. In this case, the problem is solved in Section 5. In what follows, suppose that $(r \phi(r))^{\prime \prime} \neq 0$ for all $r>0$. Then the first characteristic field is genuinely nonlinear. Let $u=r \theta$ with $\theta=\left(\theta_{1}, \theta_{2}\right)^{t} \in S^{1}$, the unit circle. For smooth solutions, the system (3.1) can be put in diagonal form :

$$
\left\{\begin{array}{l}
\partial_{t} r+(r \phi(r))^{\prime} \partial_{x} r=0 \\
\partial_{t} \theta+\phi(r) \partial_{x} \theta=0, \quad t>0, x \in \mathbb{R} .
\end{array}\right.
$$

Hence, it is hyperbolic but not strictly hyperbolic since it may occur $\phi(r)=(r \phi(r))^{\prime}$, i.e., $\phi^{\prime}(r)=0$ for some $r>0$. For the Cauchy problem (3.1)-(3.2) we refer the reader to $[13,14,23,28,31,40]$ and the references therein. When the initial data satisfy $u^{0} \in$ $B V(\mathbb{R})$, the existence of entropy solutions can be proved by using the Glimm scheme [27]. This is partially due to the decoupling of the system which compensates the loss of non strict hyperbolicity. The entropy solutions in $L^{\infty}(\mathbb{R})$ is investigated in [14].

The two families of entropy-entropy flux pairs $\left(E_{i}(u), F_{i}(u)\right)_{1 \leq i \leq 2}$ are

$$
E_{1}(u)=g_{1}(r), \quad F_{1}(u)=\int g_{1}^{\prime}(r)(r \phi(r))^{\prime} d r
$$

and

$$
E_{2}(u)=r g_{2}(\theta), \quad F_{2}(u)=r \phi(r) g_{2}(\theta),
$$


where $g_{1}$ and $g_{2}$ are smooth functions. By checking the Rankine-Hugoniot conditions of the system, we see that the entropy-entropy flux pairs $\left(E_{1}, F_{1}\right)$ and $\left(E_{2}, F_{2}\right)$ correspond to the genuinely nonlinear field $\lambda_{1}(u)$ and the linearly degenerate field $\lambda_{2}(u)$, respectively. Therefore, we require that any entropy solution $u$ of (3.1) satisfies :

$$
\partial_{t}\left(r g_{2}(\theta)\right)+\partial_{x}\left(r \phi(r) g_{2}(\theta)\right)=0,
$$

in the sense of distributions for any continuous function $g_{2}$. Let $g_{2}(\theta)=1$ and $g_{2}(\theta)=$ $\theta_{i}(i=1,2)$. We obtain

$$
\left\{\begin{array}{l}
\partial_{t} r+\partial_{x}(r \phi(r))=0 \\
\partial_{t}(r \theta)+\partial_{x}(r \phi(r) \theta)=0, \quad t>0, x \in \mathbb{R} .
\end{array}\right.
$$

It is clear that (3.1) is not a linear Lagrangian system (see definition 4.1). However, it is equivalent to (3.5) for weak solutions satisfying (3.4). Since the system (3.5) is decoupled, we may first solve the scalar conservation law for $r$ then find $\theta$ by an E-L transformation.

More precisely, assume $u^{0} \in L^{\infty}(\mathbb{R})$ and $\inf _{x \in \mathbb{R}} r^{0}(x) \geq \underline{r}>0$ with $r^{0}=\left|u^{0}\right|$. Let $r$ be the unique entropy solution of the first equation in (3.5) with initial datum $r^{0}$. It satisfies the entropy inequality

$$
\partial_{t} g_{1}(r)+\partial_{x} \int g_{1}^{\prime}(r)(r \phi(r))^{\prime} d r \leq 0, \quad \forall g_{1} \text { convex }
$$

in the sense of distributions. From the maximal principle, we know that $r \in L^{\infty}\left(\mathbb{R}^{+} \times \mathbb{R}\right)$ and $r(t, x) \geq \underline{r}$, a.e., $(t, x) \in \mathbb{R}^{+} \times \mathbb{R}$. Let $(t, x) \longmapsto(s, y)=(t, Y(t, x))$ be the unique E-L transformation defined by

$$
d y=r d x-r \phi(r) d t, \quad Y(0, x)=Y_{0}(x) \stackrel{\text { def }}{=} \int_{0}^{x} r^{0}(\xi) d \xi .
$$

Then

$$
Y(t, x)=Y_{0}(x)-\int_{0}^{t}(r \phi(r))(\tau, x) d \tau
$$

In Lagrangian coordinates $(s, y)$, the second equation in (3.5) becomes :

$$
\partial_{s} \tilde{\theta}(s, y)=0, \quad s>0, y \in \mathbb{R} .
$$

From Theorem 2.1 we obtain a unique solution $\theta$ with initial datum $\theta^{0}=u^{0} / r^{0}$, given by

$$
\theta(t, x)=\theta^{0}\left(X_{0}(Y(t, x))\right), \quad \text { with } X_{0}=Y_{0}^{-1} .
$$

When the function $\psi(r)=r \phi(r)$ is strictly convex, $Y$ can be expressed explicitly by using the conjugated function due to the Lax formula [29] :

$$
Y(t, x)=\inf _{\xi \in \mathbb{R}}\left(Y_{0}(\xi)+t \psi^{*}\left(\frac{x-\xi}{t}\right)\right), \quad \psi^{*}(y)=\sup _{\eta \in \mathbb{R}}(\eta y-\psi(\eta)) .
$$

Theorem 3.1. Assume $u^{0} \in L^{\infty}(\mathbb{R})$ with $\inf _{x \in \mathbb{R}} r^{0}(x)>0$. Then the Cauchy problem (3.1)-(3.2) has a unique global entropy solution $u=r \theta$ satisfying the entropy equality (3.4) and entropy inequality (3.6) for all continuous functions $g_{1}$ and $g_{2}$ with $g_{1}$ convex. Moreover, if the function $\psi(r)=r \phi(r)$ is strictly convex for $r>0$, this solution is given explicitly by $r=\partial_{x} Y$ and (3.8), where $Y$ is defined in (3.9). 


\section{StUdy OF LINEAR LAGRANGIAN SYSTEMS}

4.1. Definition and structure of the system. Let us begin with a definition.

Definition 4.1. A system of conservation laws (2.1) is called a linear Lagrangian one if it is linear in Lagrangian coordinates $(s, y)$ defined by the E-L transformation (2.4).

As we will see in the next sections, the class of linear Lagrangian systems contains many interesting examples such as the equations of pressureless gas dynamics, all $2 \times 2$ linear degenerate systems of conservation laws and the augmented Born-Infeld equations. By Proposition 2.1, it is easy to see that if a linear Lagrangian system is hyperbolic then it is necessarily linearly degenerate and admits a complete set of classical Riemann invariants. Moreover, since $\tilde{\lambda}_{i}$ is a constant, from (2.11) the multiplicity of each eigenvalue of a linear Lagrangian system is constant.

Now let us look at the structure of this system. The conservative variables of the system (2.5) is $v=\left(v_{1}, v_{2}, \cdots, v_{n}\right)^{t}$ defined in (2.7). Therefore, the system (2.5) is linear if and only if there are real constants $\tilde{a}_{i j}(1 \leq i, j \leq n)$ such that

$$
-\frac{f_{1}(u)}{u_{1}}=\sum_{j=1}^{n} \tilde{a}_{1 j} v_{j}, \quad f_{i}(u)-\frac{f_{1}(u) u_{i}}{u_{1}}=\sum_{j=1}^{n} \tilde{a}_{i j} v_{j} \quad(2 \leq i \leq n),
$$

which yield

$$
\left\{\begin{array}{l}
f_{1}(u)=-\tilde{a}_{11}-\sum_{j=2}^{n} \tilde{a}_{1 j} u_{j}, \\
f_{i}(u)=\frac{1}{u_{1}}\left(\tilde{a}_{i 1}+u_{i} f_{1}(u)+\sum_{j=2}^{n} \tilde{a}_{i j} u_{j}\right) \quad(2 \leq i \leq n) .
\end{array}\right.
$$

Hence, a linear Lagrangian system is necessarily of the form :

$$
\left\{\begin{array}{l}
\partial_{t} u_{1}-\partial_{x}\left(\sum_{j=2}^{n} \tilde{a}_{1 j} u_{j}\right)=0, \\
\partial_{t} u_{i}+\partial_{x}\left[\frac{1}{u_{1}}\left(\tilde{a}_{i 1}+u_{i} f_{1}(u)+\sum_{j=2}^{n} \tilde{a}_{i j} u_{j}\right)\right]=0 \quad(2 \leq i \leq n) .
\end{array}\right.
$$

It can be written as a linear system in Lagrangian coordinates :

$$
\partial_{s} \tilde{v}+\tilde{A} \partial_{y} \tilde{v}=0
$$

where $\tilde{A}=\left(\tilde{a}_{i j}\right)_{1 \leq i, j \leq n}$ is a constant matrix. Thus a linear Lagrangian system is completely determined by a constant matrix. We remark that $f_{1}$ is an affine function of $u$ and the system (4.2) can be rewritten in an equivalent form with the variables $\left(u_{1}, v_{2}, \cdots, v_{n}\right)^{t}$ :

$$
\left\{\begin{array}{l}
\partial_{t} u_{1}-\partial_{x}\left(u_{1} \sum_{j=2}^{n} \tilde{a}_{1 j} v_{j}\right)=0, \\
\partial_{t}\left(u_{1} v_{i}\right)+\partial_{x}\left(\frac{\tilde{a}_{i 1}}{u_{1}}-\tilde{a}_{11} v_{i}+\sum_{j=2}^{n}\left(\tilde{a}_{i j}-\tilde{a}_{1 j} u_{1} v_{i}\right) v_{j}\right)=0 \quad(2 \leq i \leq n) .
\end{array}\right.
$$

In what follows we suppose that the linear Lagrangian system (4.2) is hyperbolic. By Proposition 2.1, the linear system (4.3) is also hyperbolic. Then $\left(\tilde{l}_{1}, \tilde{l}_{2}, \cdots, \tilde{l}_{n}\right)$ is a basis 
of $\mathbb{R}^{n}$ with $\tilde{l}_{i}$ being an $i$-th left eigenvector of $\tilde{A}$ associated to $\tilde{\lambda}_{i}$. It follows that $w_{i}=\tilde{l}_{i} v$ is an $i$-th classical Riemann invariant of (4.2) and satisfies

$$
\partial_{s} \tilde{w}_{i}+\tilde{\lambda}_{i} \partial_{y} \tilde{w}_{i}=0 \quad(1 \leq i \leq n) .
$$

Therefore, each entropy-entropy flux pair $(\tilde{E}, \tilde{F})$ of the system $(4.3)$ has the form :

$$
\left\{\begin{array}{l}
\tilde{E}(v)=\sum_{j=1}^{n} g_{j}\left(w_{j}\right)=\sum_{j=1}^{n} g_{j}\left(\tilde{l}_{j} v\right), \\
\tilde{F}(v)=\sum_{j=1}^{n} \tilde{\lambda}_{j} g_{j}\left(w_{j}\right)=\sum_{j=1}^{n} \tilde{\lambda}_{j} g_{j}\left(\tilde{l}_{j} v\right),
\end{array}\right.
$$

where $g_{j}(1 \leq j \leq n)$ are arbitrary continuous functions. From the equivalence relation (2.6), we obtain an explicit expression of each entropy-entropy flux pair of the linear Lagrangian system as :

$$
(E(u), F(u))=\sum_{j=1}^{n}\left(u_{1}, \tilde{\lambda}_{j}+f_{1}(u)\right) g_{j}\left(\frac{1}{u_{1}} \tilde{l}_{j}\left(1, u_{2}, \cdots, u_{n}\right)^{t}\right) .
$$

Moreover, let $\tilde{l}_{i j}$ be the $i$-th component of $\tilde{l}_{j}$, we have

$$
\frac{\partial^{2} \tilde{E}(v)}{\partial v_{i} \partial v_{k}}=\sum_{j=1}^{n} g_{j}^{\prime \prime}\left(w_{j}\right) \tilde{l}_{j i} \tilde{l}_{j k} \stackrel{\text { def }}{=} \alpha_{i k}^{g}(w), \quad \text { with } w=\left(w_{1}, \cdots, w_{n}\right)^{t} .
$$

Therefore, by the equivalence of the convexity of entropies given in Proposition 2.1, $E(u)$ defined by (4.7) is convex if and only if the symmetric matrix $\Delta_{g}(w) \stackrel{\text { def }}{=}\left(\alpha_{i k}^{g}(w)\right)_{1 \leq i, k \leq n}$ is positively definite for all $w \in \mathbb{R}^{n}$. In particular, since $\left(\tilde{l}_{1}, \tilde{l}_{2}, \cdots, \tilde{l}_{n}\right)$ is linearly independent, the constant matrix $\left(<\tilde{l}_{i}, \tilde{l}_{k}>\right)_{1 \leq i, k \leq n}$ is positively definite, where $<\cdot, \cdot>$ denotes the inner product of $\mathbb{R}^{n}$. Thus a strictly convex entropy $E(u)$ is constructed by choosing $g_{j}(\xi)=\xi^{2}$ for all $j=1,2, \cdots, n$.

We collect the above discussion in the following result.

Proposition 4.1. Suppose that the linear Lagrangian system (4.2) is hyperbolic. Then it is linearly degenerate, admits a complete set of classical Riemann invariants defined by $w_{i}=\tilde{l}_{i}\left(1, u_{2}, \cdots, u_{n}\right)^{t} / u_{1}(1 \leq i \leq n)$ and each of its eigenvalues has constant multiplicity, where $\left(\tilde{l}_{1}, \tilde{l}_{2}, \cdots, \tilde{l}_{n}\right)$ is composed of the left eigenvectors of the corresponding linear system in Lagrangian coordinates. Moreover, each entropy-entropy flux pair $(E(u), F(u))$ of the system has an explicit expression (4.7), E(u) is convex if and only if the matrix $\Delta_{g}(w)$ is positively definite for all $w \in \mathbb{R}^{n}$ and a strictly convex entropy $E(u)$ is given when $g_{j}(\xi)=\xi^{2}$ for all $j=1,2, \cdots, n$.

Finally, we mention that a similar notion was introduced in [19] where a system of conservation laws is called a Lagrangian system if in Lagrangian coordinates it admits a strictly convex entropy with zero flux function. The Lagrangian system is an extension of the equations for gas dynamics. Then the nature of the linear Lagrangian system and Lagrangian system is different.

4.2. Explicit solutions of the Cauchy problem. Consider the Cauchy problem for the linear Lagrangian system (4.2) with the initial condition (2.2). Let $v^{0}=\left(1, u_{2}^{0}, \cdots, u_{n}^{0}\right)^{t} / u_{1}^{0}$ and $w=\left(w_{1}, w_{2}, \cdots, w_{n}\right)^{t}$. By Theorem 2.1 we have

$$
s=0: \quad \tilde{w}_{i}=w_{i}^{0}\left(X_{0}(y)\right)=\tilde{l}_{i} v^{0}\left(X_{0}(y)\right), \quad y \in \mathbb{R} .
$$


Therefore, the unique solution of the Cauchy problem (4.3) and (4.8) is given by

$$
\tilde{w}_{i}(s, y)=w_{i}^{0}\left(X_{0}\left(y-\tilde{\lambda}_{i} s\right)\right) .
$$

It follows from Theorem 2.1 that the Cauchy problem (4.2) and (2.2) admits a unique entropy solution $u$. For seeking an explicit expression of $u$, we denote by $\tilde{r}_{i}$ the right eigenvector of $\tilde{A}$ associated to $\tilde{\lambda}_{i}$ normalized as $\tilde{l}_{i} \tilde{r}_{j}=\delta_{i j}$, where $\delta_{i j}$ stands for the Kronecker's symbol. Then we may write

$$
\tilde{v}(s, y)=\sum_{i=1}^{n}\left(\tilde{l}_{i} v^{0}\right)\left(X_{0}\left(y-\tilde{\lambda}_{i} s\right)\right) \tilde{r}_{i} .
$$

Now let us determine the unique function $y=Y(t, x)$ in the E-L transformation. From (2.4), we have

$$
d x=\tilde{v}_{1}(t, y) d y-\sum_{j=1}^{n} \tilde{a}_{1 j} \tilde{v}_{j}(t, y) d t
$$

which defines a unique function $x=X(t, y)$ together with $X(0, y)=X_{0}(y)$. A straightforward calculation using the first equation in (4.3) gives

$$
X(t, y)=X_{0}(y)-\int_{0}^{t} \sum_{j=1}^{n} \tilde{a}_{1 j} \tilde{v}_{j}(\tau, y) d \tau
$$

Let $\tilde{r}_{j i}$ be the $j$-th component of $\tilde{r}_{i}$. Since

$$
\sum_{j=1}^{n} \tilde{a}_{1 j} \tilde{r}_{j i}=\tilde{\lambda}_{i} \tilde{r}_{1 i}
$$

it follows from (4.9) that

$$
X(t, y)=X_{0}(y)-\int_{0}^{t} \sum_{i=1}^{n} \tilde{\lambda}_{i} \tilde{r}_{1 i}\left(\tilde{l}_{i} v^{0}\right)\left(X_{0}\left(y-\tilde{\lambda}_{i} \tau\right)\right) d \tau .
$$

Note that the function in the above integral is given by the initial data. To guarantee that the function $y \longmapsto X(t, y)$ is invertible for all $t \geq 0$, we need the conditions $v \in$ $L^{\infty}\left(\mathbb{R}^{+} \times \mathbb{R}\right)$ and $v_{1}(t, x) \geq \underline{v_{1}}>0$, or equivalently $u \in L^{\infty}\left(\mathbb{R}^{+} \times \mathbb{R}\right)$ and $u_{1}(t, x) \geq \underline{u_{1}}>0$, a.e. $(t, x) \in \mathbb{R}^{+} \times \mathbb{R}$. That is why we have made the assumptions

$$
u^{0} \in L^{\infty}(\mathbb{R}), \quad u_{1}^{0}(x) \geq \underline{u_{1}}>0, \quad \text { a.e. } x \in \mathbb{R}
$$

and furthermore,

$$
\sum_{i=1}^{n}\left(\tilde{l}_{i} v^{0}\right)\left(X_{0}\left(y-\tilde{\lambda}_{i} t\right)\right) \tilde{r}_{1 i} \geq \underline{v_{1}}>0, \quad \text { a.e. }(t, y) \in \mathbb{R}^{+} \times \mathbb{R} .
$$

It is clear that under the conditions (4.11)-(4.12), the function $y \longmapsto X(t, y)$ is invertible and bi-Lipschitzian from $\mathbb{R}$ to $\mathbb{R}$ for all $t \geq 0$. Therefore, from Lemma 2.1 we obtain $y=Y(t, x)$ as the inverse function of $y \longmapsto X(t, y)$ satisfying $Y(0, x)=Y_{0}(x)$. Finally, (4.9) gives

$$
v(t, x)=\sum_{i=1}^{n}\left(\tilde{l}_{i} v^{0}\right)\left(X_{0}\left(Y(t, x)-\tilde{\lambda}_{i} t\right)\right) \tilde{r}_{i}
$$


which implies, together with (4.12), that $v_{1}(t, x) \geq v_{1}>0$, a.e. $(t, x) \in \mathbb{R}^{+} \times \mathbb{R}$. In particular,

$$
w_{i}(t, x)=w_{i}^{0}\left(X_{0}\left(Y(t, x)-\tilde{\lambda}_{i} t\right)\right),
$$

which shows that the maximum principle holds for each $w_{i}$.

On the other hand, the expression $\tilde{w}_{i}(s, y)=\tilde{w}_{i}^{0}\left(y-\tilde{\lambda}_{i} s\right)$ shows that the entropy equality (2.10) is satisfied for all entropy-entropy flux pairs $(\tilde{E}(v), \tilde{F}(v))$ given in (4.6). This equality is equivalent to (2.9). Thus we obtain the following conclusion.

Theorem 4.1. Let the assumptions (4.11)-(4.12) hold and the linear Lagrangian system (4.2) be hyperbolic. Then the Cauchy problem (4.2) and (2.2) admits a unique entropy solution in the sense of Definition 2.1. It is given by the explicit expression (4.13) and (2.7), where $X_{0}$ and $Y(t, \cdot)$ are the inverse functions of $Y_{0}$ and $X(t, \cdot)$ for all $t \geq 0$ defined in (2.12) and (4.10), respectively.

Remark 4.1. Formula (4.13) shows that the solution of the Cauchy problem (4.2) and (2.2) has the same regularity as its initial data. In particular, if $u^{0} \in B V(\mathbb{R})$ then $u(t, \cdot) \in B V(\mathbb{R})$ for all $t \geq 0$; and if $u^{0} \in C^{m}(\mathbb{R})$ with $m \in \mathbb{N}^{*}$ then $X_{0}, Y_{0} \in C^{m}(\mathbb{R})$ and $X, Y \in C^{m}\left(\mathbb{R}^{+} \times \mathbb{R}\right)$. Hence, $u \in C^{m}\left(\mathbb{R}^{+} \times \mathbb{R}\right)$.

Remark 4.2. From (4.10), we have

$$
\partial_{t t} X(t, y)=\sum_{i=1}^{n} \tilde{\lambda}_{i}^{2} \tilde{r}_{1 i} \tilde{l}_{i}\left(v^{0} \circ X_{0}\right)^{\prime}\left(y-\tilde{\lambda}_{i} t\right)
$$

and

$$
\partial_{y y} X(t, y)=\sum_{i=1}^{n} \tilde{r}_{1 i} \tilde{l}_{i}\left(v^{0} \circ X_{0}\right)^{\prime}\left(y-\tilde{\lambda}_{i} t\right) .
$$

Hence, when $\left|\tilde{\lambda}_{i}\right|=\tilde{\lambda}$ for all $i=1,2, \cdots, n, X$ satisfies a linear wave equation

$$
\partial_{t t} X-\tilde{\lambda}^{2} \partial_{y y} X=0 \text {. }
$$

As we will see in the next section, this situation occurs for all $2 \times 2$ linearly degenerate systems of conservation laws.

4.3. Weak stability. Consider a hyperbolic linear Lagrangian system (4.2). Let $\left(u_{\varepsilon}^{0}\right)_{\varepsilon>0}$ be a bounded sequence in $L^{\infty}(\mathbb{R})$ satisfying (4.11)-(4.12) uniformly with respect to $\varepsilon$. We denote by $\left(u_{\varepsilon}\right)_{\varepsilon>0}$ the corresponding sequence of entropy solutions of the system (4.2) with the initial data $\left(u_{\varepsilon}^{0}\right)_{\varepsilon>0}$. From (4.11)-(4.12) and the explicit formulas (4.13)-(4.14), we know that the sequence $\left(u_{\varepsilon}\right)_{\varepsilon>0}$ is bounded in $L^{\infty}\left(\mathbb{R}^{+} \times \mathbb{R}\right)$ and there is a constant $\underline{u}>0$, independent of $\varepsilon \rightarrow 0$, such that $u_{1 \varepsilon}(t, x) \geq \underline{u}$, a.e. $(t, x) \in \mathbb{R}^{+} \times \mathbb{R}$.

Therefore, up to subsequences, still denoted by $\left(u_{\varepsilon}\right)_{\varepsilon>0}$ and $\left(u_{\varepsilon}^{0}\right)_{\varepsilon>0}$, we have

$$
u_{\varepsilon} \longrightarrow u, \quad \text { in } L^{\infty}\left(\mathbb{R}^{+} \times \mathbb{R}\right) \text { weakly-* }
$$

and

$$
u_{\varepsilon}^{0} \longrightarrow u^{0}, \quad \text { in } L^{\infty}(\mathbb{R}) \text { weakly-* },
$$

with $u \in L^{\infty}\left(\mathbb{R}^{+} \times \mathbb{R}\right)$ and $u^{0} \in L^{\infty}(\mathbb{R})$ satisfying $u_{1}^{0}(x) \geq \underline{u_{1}}>0$ and $u_{1}(t, x) \geq \underline{u}$, a.e. $(t, x) \in \mathbb{R}^{+} \times \mathbb{R}$. The weak stability of the linear Lagrangian system means that the weak limit $u$ is a unique entropy solution of (4.2) with initial datum $u^{0}$. 
Indeed, since $f_{1}$ is an affine function, we obtain

$$
f_{1}\left(u_{\varepsilon}\right) \longrightarrow f_{1}(u), \quad \text { in } L^{\infty}\left(\mathbb{R}^{+} \times \mathbb{R}\right) \text { weakly- } * .
$$

Then applying the div-curl lemma of compensated compactness (see [43]) to the first and $i$-th $(2 \leq i \leq n)$ equations in (4.2), we obtain, in the sense of distributions,

$$
\lim _{\varepsilon \rightarrow 0}\left(u_{1 \varepsilon} f_{i}\left(u_{\varepsilon}\right)-u_{i \varepsilon} f_{1}\left(u_{\varepsilon}\right)\right)=u_{1} \lim _{\varepsilon \rightarrow 0} f_{i}\left(u_{\varepsilon}\right)-u_{i} \lim _{\varepsilon \rightarrow 0} f_{1}\left(u_{\varepsilon}\right) .
$$

It follows from the definition of $f_{i}$ that

$$
\lim _{\varepsilon \rightarrow 0}\left(\tilde{a}_{i 1}+\sum_{j=2}^{n} \tilde{a}_{i j} u_{j \varepsilon}\right)=u_{1} \lim _{\varepsilon \rightarrow 0} f_{i}\left(u_{\varepsilon}\right)-u_{i} f_{1}(u) .
$$

Hence, for all $2 \leq i \leq n$,

$$
\lim _{\varepsilon \rightarrow 0} f_{i}\left(u_{\varepsilon}\right)=\frac{1}{u_{1}}\left(\tilde{a}_{i 1}+u_{i} f_{1}(u)+\sum_{j=2}^{n} \tilde{a}_{i j} u_{j}\right)=f_{i}(u) .
$$

This allows to pass to the limits in the weak formulation of the Cauchy problem for $u_{\varepsilon}$ and deduce that $u$ is a weak solution of (4.2) and the initial datum of $u$ is just $u^{0}$.

To prove that the weak limit $u$ is a unique entropy solution of $(4.2)$, it remains to show that $u$ fulfills the condition (4.12). To this end, we notice that the sequence $\left(Y_{0 \varepsilon}\right)_{\varepsilon>0}$ defined by

$$
Y_{0 \varepsilon}(x)=\int_{0}^{x} u_{1 \varepsilon}^{0}(\xi) d \xi
$$

is bounded in $W_{l o c}^{1, \infty}(\mathbb{R})$. Then up to a subsequence, still denoted by $\left(Y_{0 \varepsilon}\right)_{\varepsilon>0}$, we have $Y_{0 \varepsilon} \longrightarrow Y_{0}$ in $L_{l o c}^{p}(\mathbb{R})$ strongly for any $p \geq 1$, where $Y_{0}$ is given in $(2.12)$. Let $\phi \in C_{0}^{\infty}(\mathbb{R})$ and $X_{0 \varepsilon}=Y_{0 \varepsilon}^{-1}$. Then $\phi\left(Y_{0 \varepsilon}\right) \longrightarrow \phi\left(Y_{0}\right)$ in $L_{l o c}^{p}(\mathbb{R})$ strongly. It follows that

$$
\begin{aligned}
\int_{\mathbb{R}}\left(v_{\varepsilon}^{0} \circ X_{0 \varepsilon}\right)(y) \phi(y) d y & =\int_{\mathbb{R}} v_{\varepsilon}^{0}(x) \phi\left(Y_{0 \varepsilon}(x)\right) Y_{0 \varepsilon}^{\prime}(x) d x \\
& =\int_{\mathbb{R}} v_{\varepsilon}^{0}(x) u_{1 \varepsilon}^{0}(x) \phi\left(Y_{0 \varepsilon}(x)\right) d x \\
& =\int_{\mathbb{R}}\left(1, u_{2 \varepsilon}^{0}, \cdots, u_{n \varepsilon}^{0}\right)^{t}(x) \phi\left(Y_{0 \varepsilon}(x)\right) d x \\
& \int_{\mathbb{R}}\left(1, u_{2}^{0}, \cdots, u_{n}^{0}\right)^{t}(x) \phi\left(Y_{0}(x)\right) d x \\
& =\int_{\mathbb{R}}\left(v^{0} \circ X_{0}\right)(y) \phi(y) d y,
\end{aligned}
$$

with $v^{0}=\left(1, u_{2}^{0}, \cdots, u_{n}^{0}\right)^{t} / u_{1}^{0}$. Here we have used the changes of variables $y=Y_{0 \varepsilon}(x)$ and $y=Y_{0}(x)$ in the first and last equalities, respectively. This shows that

$$
v_{\varepsilon}^{0} \circ X_{0 \varepsilon} \longrightarrow v^{0} \circ X_{0}, \quad \text { in } L^{\infty}(\mathbb{R}) \text { weakly- * . }
$$

Since $u_{\varepsilon}^{0}$ satisfies (4.12) uniformly with respect to $\varepsilon$, i.e.,

$$
\sum_{i=1}^{n}\left(\tilde{l}_{i} v_{\varepsilon}^{0}\right)\left(X_{0 \varepsilon}\left(y-\tilde{\lambda}_{i} t\right)\right) \tilde{r}_{1 i} \geq \underline{v_{1}}>0, \quad \text { a.e. }(t, y) \in \mathbb{R}^{+} \times \mathbb{R},
$$


we deduce that $u$ satisfies (4.12). Thus, $u$ is a unique entropy solution of the Cauchy problem to the linear Lagrangian system (4.2).

Theorem 4.2. The linear Lagrangian system is weakly stable in $L^{\infty}\left(\mathbb{R}^{+} \times \mathbb{R}\right)$.

Similar results were obtained by Serre [38] for $2 \times 2$ linearly degenerate systems and by Brenier [9] for a simplified ABI system. Since these two systems are included in the class of linear Lagrangian systems, Theorem 4.2 can be viewed as an extension of their results. However, the entropy equality and uniqueness of entropy solutions were not discussed in their papers. Finally, we point out that the above weak stability result is obtained in a very simple way with the help of a good choice of conservation laws to apply the div-curl lemma among all entropy-entropy flux pairs given in (4.7).

4.4. An example of non-hyperbolic linear Lagrangian systems. Consider the Cauchy problem for the system of pressureless gas dynamics :

$$
\left\{\begin{array}{l}
\partial_{t} \rho+\partial_{x}(\rho u)=0, \\
\partial_{t}(\rho u)+\partial_{x}\left(\rho u^{2}\right)=0, \quad t>0, x \in \mathbb{R},
\end{array}\right.
$$

with initial conditions :

$$
t=0: \rho=\rho^{0}(x), u=u^{0}(x), \quad x \in \mathbb{R} .
$$

Let $\rho>0$ and $(t, x) \longmapsto(s, y)=(t, Y(t, x))$ be the E-L transformation defined by

$$
d y=\rho d x-\rho u d t, \quad Y(0, x)=Y_{0}(x) \stackrel{\text { def }}{=} \int_{0}^{x} \rho^{0}(\xi) d \xi .
$$

Then in Lagrangian coordinates $(s, y)$, the system (4.15) becomes :

$$
\left\{\begin{array}{l}
\partial_{s} \tilde{v}-\partial_{y} \tilde{u}=0, \\
\partial_{s} \tilde{u}=0, \quad s>0, y \in \mathbb{R} .
\end{array}\right.
$$

It is clear that the equations of pressureless gas dynamics are linear Lagrangian system but not hyperbolic.

The initial conditions (4.16) correspond to :

$$
s=0: \tilde{v}=\tilde{v}^{0}(y), \tilde{u}=\tilde{u}^{0}(y), \quad y \in \mathbb{R},
$$

where

$$
\left(\tilde{v}^{0}, \tilde{u}^{0}\right)=\left(\frac{1}{\rho^{0}}, u^{0}\right)\left(X_{0}\right), \quad X_{0}=Y_{0}^{-1} .
$$

We deduce from (4.18)-(4.19) that

$$
\tilde{v}(t, y)=\tilde{v}^{0}(y)+t\left(\tilde{u}^{0}\right)^{\prime}(y), \quad \tilde{u}(t, y)=\tilde{u}^{0}(y) .
$$

To guarantee the change of variables (4.17), we assume that

$$
\rho^{0} \in L^{\infty}(\mathbb{R}), \quad u^{0} \in W^{1, \infty}(\mathbb{R}) \text { with } \rho^{0}(x) \geq \underline{\rho}>0, \text { a.e. } x \in \mathbb{R} .
$$

Then $\tilde{v}(t, y)>0$ for small $t>0$. It follows that $x=X(t, y)$ satisfies

$$
d x=\left(\tilde{v}^{0}(y)+t\left(\tilde{u}^{0}\right)^{\prime}(y)\right) d y+\tilde{u}^{0}(y) d t .
$$

The unique function $x=X(t, y)$ satisfying $X(0, y)=X_{0}(y)=Y_{0}^{-1}(y)$ is

$$
X(t, y)=X_{0}(y)+t \tilde{u}^{0}(y) .
$$


Since $X_{0}^{\prime}(y)=\tilde{v}^{0}(y)$, the function $y \longmapsto X(t, y)$ is bijective and bi-Lipschitzian for small $t \geq 0$. From Lemma 2.1 , we have $Y(t, \cdot)=X^{-1}(t, \cdot)$ for small $t \geq 0$. Hence,

$$
\rho(t, x)=\frac{\rho^{0}\left(X_{0}(Y(t, x))\right)}{1+t\left(\rho^{0}\left(u^{0}\right)^{\prime}\right)\left(X_{0}(Y(t, x))\right)}, \quad u(t, x)=u^{0}\left(X_{0}(Y(t, x))\right) .
$$

Proposition 4.2. Under the assumption (4.20), there exists $t_{1}>0$ such that the Cauchy problem (4.15)-(4.16) admits a unique solution $(\rho, u) \in L^{\infty}\left(\left(0, t_{1}\right) \times \mathbb{R}\right) \cap W^{1, \infty}\left(\left(0, t_{1}\right) \times \mathbb{R}\right)$, defined by (4.21). This solution is global if $u^{0}$ is a non-decreasing function.

Beyond the interval $\left[0, t_{1}\right), \rho$ becomes a measure. For the study of measure-valued solutions of (4.15), we refer to $[6,7,8,22]$ and the references therein. See also [47] from a physical point of view.

\section{The $2 \times 2$ Linearly DEgenerate SyStem}

Consider the Cauchy problem for the $2 \times 2$ linearly degenerate system. Since the characteristic fields are both linearly degenerate, the eigenvalues $\lambda_{1}$ and $\lambda_{2}$ are two classical Riemann invariants of the system associated to the eigenvalues $\lambda_{2}$ and $\lambda_{1}$, respectively. Set $z=\lambda_{1}$ and $w=\lambda_{2}$, then for smooth solutions all $2 \times 2$ linearly degenerate systems can be written in a canonical form :

$$
\left\{\begin{array}{l}
\partial_{t} w+z \partial_{x} w=0 \\
\partial_{t} z+w \partial_{x} z=0, \quad t>0, x \in \mathbb{R} .
\end{array}\right.
$$

It is supplemented by initial conditions :

$$
t=0: w=w^{0}(x), z=z^{0}(x), \quad x \in \mathbb{R} .
$$

It is easy to check that each entropy-entropy flux pair $(E, F)$ of the system (5.1) is of the form (see [38]) :

$$
E(w, z)=\frac{g_{1}(w)+g_{2}(z)}{w-z}, \quad F(w, z)=\frac{z g_{1}(w)+w g_{2}(z)}{w-z},
$$

where $g_{1}$ and $g_{2}$ are two arbitrary continuous functions.

For $w>z$ and any $a>0$, let

$$
\rho=\frac{2 a}{w-z}, \quad u=\frac{w+z}{2} .
$$

Then we obtain the equations for Chaplygin gas dynamics [46] :

$$
\left\{\begin{array}{l}
\partial_{t} \rho+\partial_{x}(\rho u)=0, \\
\partial_{t}(\rho u)+\partial_{x}\left(\rho u^{2}+p(\rho)\right)=0,
\end{array}\right.
$$

in which the pressure is given by the state law of Von Kármán-Tsien $: p(\rho)=p^{\prime}-a^{2} \rho^{-1}$, where $p^{\prime}>0$ is a constant such that $p(\rho)>0$ (see [15], p.10 and [2], p.7).

Comparing the systems (4.4) and (5.5), we see that (5.5) is a linear Lagrangian system with $n=2, u_{1}=\rho$ and $u_{2}=\rho u$. According to Definition $2.1,(w, z) \in L^{\infty}\left(\mathbb{R}^{+} \times \mathbb{R}\right)$ with $w>z$ is an entropy solution of the Cauchy problem (5.1)-(5.2) if all entropy-entropy flux pairs $(E(w, z), F(w, z))$ defined by (5.3) satisfy

$$
\partial_{t} E(w, z)+\partial_{x} F(w, z)=0
$$


in the sense of distributions. This makes sense of the system (5.1) for weak solutions.

The system (5.1) was studied by Serre for oscillatory solutions (see [38] or [40], p.110113), and by E and Kohn for measure-valued solutions [21]. When the initial data satisfy

$$
w^{0}, z^{0} \in L^{\infty}(\mathbb{R}), \inf _{x \in \mathbb{R}} w^{0}(x)>\sup _{x \in \mathbb{R}} z^{0}(x),
$$

the existence and uniqueness of entropy solutions were established by Chen by using the compensated compactness and quasidecoupling method [14]. The derivation of explicit solutions of the Cauchy problem (5.1)-(5.2) was investigated by Serre [39] and by Brenier $[9,10]$ in the case of periodic smooth solutions.

Under the conditions in (5.7), the explicit entropy solution in $L^{\infty}$ of the Cauchy problem was constructed by the author [36] by means of the method presented in Sections 2-4. The resulting formula is the same as that in $[9,10]$ but is different from that of [39]. Indeed, it is a particular case of the linear Lagrangian system for which the second condition in (5.7) corresponds to (4.12). This condition implies that $w(t, x)>z(t, x)$ and then the system (5.1) is strictly hyperbolic for almost all $(t, x) \in \mathbb{R}^{+} \times \mathbb{R}$.

The E-L transformation can be defined explicitly by using the first equation in $(5.5)$ and (5.4) with $a=1$. It is given by $(s, y)=(t, Y(t, x))$ with

$$
d y=\frac{2}{w-z} d x-\frac{w+z}{w-z} d t, \quad Y(0, x)=Y_{0}(x) \stackrel{\text { def }}{=} \int_{0}^{x} \frac{2 d \xi}{w^{0}(\xi)-z^{0}(\xi)} .
$$

The corresponding linear system in Lagrangian coordinates $(s, y)$ is

$$
\partial_{s} \tilde{w}-\partial_{y} \tilde{w}=0, \quad \partial_{s} \tilde{z}+\partial_{y} \tilde{z}=0 .
$$

Let $X_{0}=Y_{0}^{-1}$. Then

$$
\tilde{w}(s, y)=w^{0}\left(X_{0}(y+s)\right) \text { and } \tilde{z}(s, y)=z^{0}\left(X_{0}(y-s)\right) .
$$

Hence, a unique function $x=X(t, y)$ satisfying $X(0, y)=X_{0}(y)$ is given by

$$
X(t, y)=\frac{1}{2} \int_{0}^{y+t} w^{0}\left(X_{0}(\xi)\right) d \xi-\frac{1}{2} \int_{0}^{y-t} z^{0}\left(X_{0}(\xi)\right) d \xi .
$$

Obviously, $X(t, \cdot)$ is bijective and Lipschitzian from $\mathbb{R}$ to $\mathbb{R}$ for all $t \geq 0$. From Lemma 2.1, we have $Y(t, \cdot)=X^{-1}(t, \cdot)$. Finally, since the eigenvalues of the linear system (5.9) are $\tilde{\lambda}_{1}=-1$ and $\tilde{\lambda}_{2}=1$. From Remark $4.2, X$ satisfies the linear wave equation :

$$
\partial_{t t} X-\partial_{y y} X=0 \text {. }
$$

As an application of Theorem 4.1, we obtain the following result already stated in [36].

Theorem 5.1. Let the conditions in (5.7) hold. Then the Cauchy problem (5.1)-(5.2) has a unique solution $(w, z) \in L^{\infty}\left(\mathbb{R}^{+} \times \mathbb{R}\right)$ satisfying the entropy equality (5.6) for all entropy-entropy flux pairs $(E, F)$ defined by (5.3). This solution is given by

$$
w(t, x)=w^{0}\left(X_{0}(Y(t, x)+t)\right), \quad z(t, x)=z^{0}\left(X_{0}(Y(t, x)-t)\right), \quad t>0, \quad x \in \mathbb{R} .
$$

Remark 5.1. In view of the expressions (5.8) and (5.11), the second condition in (5.7) can be replaced by the following weak condition:

$$
\inf _{t>0, y \in \mathbb{R}}\left(w^{0}\left(X_{0}(y+t)\right)-z^{0}\left(X_{0}((y-t))\right)>0 .\right.
$$

By the expression (5.3) on the entropy-entropy flux pairs, it is easy to see that this weak condition is necessary and sufficient to define any weak solution. 
Remark 5.2. In the literature there exists an another way to integrate the system (5.1). Consider the second order nonlinear partial differential equation

$$
\left(\left(\partial_{x} \Phi\right)^{2}+1\right) \partial_{t t} \Phi-2 \partial_{t} \Phi \partial_{x} \Phi \partial_{t x} \Phi+\left(\left(\partial_{t} \Phi\right)^{2}-1\right) \partial_{x x} \Phi=0, \quad t>0, x \in \mathbb{R}
$$

with initial conditions

$$
t=0: \Phi=\Phi_{0}(x), \quad \partial_{t} \Phi=\Phi_{1}(x), \quad x \in \mathbb{R} .
$$

Under the hyperbolic condition $1+\left(\partial_{x} \Phi\right)^{2}-\left(\partial_{t} \Phi\right)^{2}>0$, Lochak obtained the following explicit formulas for smooth solutions of (5.12)-(5.13), see [32, 33],

$$
\begin{gathered}
t=\frac{1}{2} \int_{\beta}^{\alpha} \frac{1+\left(\Phi_{0}^{\prime}(\lambda)\right)^{2}}{\sqrt{1+\left(\Phi_{0}^{\prime}(\lambda)\right)^{2}-\Phi_{1}^{2}(\lambda)}} d \lambda, \\
x=\frac{\alpha+\beta}{2}+\frac{1}{2} \int_{\beta}^{\alpha} \frac{\Phi_{0}^{\prime}(\lambda) \Phi_{1}(\lambda)}{\sqrt{1+\left(\Phi_{0}^{\prime}(\lambda)\right)^{2}-\Phi_{1}^{2}(\lambda)}} d \lambda, \\
\Phi=\frac{\Phi_{0}(\alpha)+\Phi_{1}(\beta)}{2}-\frac{1}{2} \int_{\beta}^{\alpha} \frac{\Phi_{1}(\lambda)}{\sqrt{1+\left(\Phi_{0}^{\prime}(\lambda)\right)^{2}-\Phi_{1}^{2}(\lambda)}} d \lambda .
\end{gathered}
$$

If we introduce $u=\partial_{t} \Phi$ and $v=\partial_{x} \Phi$, then (5.12) can be rewritten as a non conservative system :

$$
\left\{\begin{array}{l}
\left(v^{2}+1\right) \partial_{t} u-2 u v \partial_{x} u+\left(u^{2}-1\right) \partial_{x} v=0 \\
\partial_{t} v-\partial_{x} u=0
\end{array}\right.
$$

This system is equivalent to (5.1) for smooth solutions in which $w$ and $z$ are defined by

$$
w=\frac{1}{v^{2}+1}\left(\sqrt{1+v^{2}-u^{2}}-u v\right), \quad z=-\frac{1}{v^{2}+1}\left(\sqrt{1+v^{2}-u^{2}}+u v\right) .
$$

It is clear that Lochak formulas (5.14)-(5.16) are different from (5.11).

\section{The Born-INFEld EQUATIONS}

6.1. Preliminary. The Born-Infeld system is one of the nonlinear models of Maxwell equations and related to the Born-Infeld Lagrangian (See Born-Infeld [5], Boillat [2], Boillat-Ruggeri [4], Brenier [9], Gibbons [25, 26] and Serre [41])

$$
L(E, B)=-\sqrt{1+|B|^{2}-|E|^{2}-<E, B>^{2}},
$$

where $E$ and $B$ are the electric and magnetic fields in $\left.\mathbb{R}^{3},<\cdot, \cdot\right\rangle$ stands for the inner product and $|B|=<B, B>^{\frac{1}{2}}$ is the Euclidean norm. Introducing the electric induction $D=\partial L / \partial E$, the Poynting vector $P$ and the Born-Infeld energy density $h[5,26,9]$ :

$$
P=D \times B, \quad h=\sqrt{1+|B|^{2}+|D|^{2}+|D \times B|^{2}},
$$

then the BI equations read :

$$
\left\{\begin{array}{l}
\partial_{t} D+\nabla \times\left(\frac{-B+D \times P}{h}\right)=0, \\
\partial_{t} B+\nabla \times\left(\frac{D+B \times P}{h}\right)=0,
\end{array}\right.
$$


with differential constraints

$$
\nabla \cdot D=\nabla \cdot B=0
$$

Remark that in the Born-Infeld model, the electric field $E \in \mathbb{R}^{3}$ is given by

$$
E=\frac{\partial h(D, B)}{\partial D}=\frac{1}{h}(D+B \times P) .
$$

It is well known that the Born-Infeld energy $h$ and Poynting vector $P$ satisfy the additional conservation laws

$$
\begin{gathered}
\partial_{t} h+\nabla \cdot P=0, \\
\partial_{t} P+\nabla \cdot\left(\frac{P \otimes P-B \otimes B-D \otimes D}{h}\right)-\nabla\left(\frac{1}{h}\right)=0,
\end{gathered}
$$

which mean that $h$ and $P$ are just mathematical entropies of the system (6.3). In [9] Brenier lifted the BI system of 6 equations (6.2)-(6.4) to the ABI system of 10 equations (6.3)-(6.6) by considering $h, D, B$ and $P$ as independent variables. He revealed the hydrodynamic structure of the ABI system similar to that of the Magnetohydrodynamics equations. An another enlargement of the BI system of 6 equations to 9 equations was described by Serre [41]. Note that the idea of enlargement of systems was developed by Qin [37], by Dafermos [17] and by Demoulini-Stuart-Tzavaras [18] for nonlinear elasticity.

For smooth solutions to the BI and ABI systems, the reader is referred to Brenier [9] and Serre [41] for local existence of smooth solutions by an argument for first order symmetrizable hyperbolic systems, to Chae-Huh [12] for global existence with small initial data, and also to [11] for the solutions beyond singularities. In this section, we consider the entropy solutions for both BI and ABI systems in one-dimensional case. In a simple way we show that both systems are hyperbolic and linearly degenerate. Therefore, $L^{\infty}$ entropy solutions of the BI system should satisfy the conservation laws (6.5)-(6.6) according to Definition 2.1. Moreover, the ABI system is a linear Lagrangian one thus allowing to apply Theorem 4.1. Although the BI system is not a linear Lagrangian one, the explicit entropy solutions can still be constructed via the solutions of the ABI system.

As in [9] we denote by $x=x_{1}$ and consider the problems independent of $x_{2}$ and $x_{3}$. Then $B_{1}$ and $D_{1}$ are constants from (6.3)-(6.4). It follows that the BI system (6.3)-(6.4) and the ABI system (6.3)-(6.6) are reduced to

$$
\left\{\begin{array}{l}
\partial_{t} D_{2}+\partial_{x}\left(\frac{B_{3}+D_{2} P_{1}-D_{1} P_{2}}{h}\right)=0, \\
\partial_{t} D_{3}+\partial_{x}\left(\frac{-B_{2}+D_{3} P_{1}-D_{1} P_{3}}{h}\right)=0, \\
\partial_{t} B_{2}+\partial_{x}\left(\frac{-D_{3}+B_{2} P_{1}-B_{1} P_{2}}{h}\right)=0, \\
\partial_{t} B_{3}+\partial_{x}\left(\frac{D_{2}+B_{3} P_{1}-B_{1} P_{3}}{h}\right)=0, \\
P=D \times B, \quad h=\sqrt{1+|B|^{2}+|D|^{2}+|D \times B|^{2}},
\end{array}\right.
$$




$$
\left\{\begin{array}{l}
\partial_{t} h+\partial_{x} P_{1}=0 \\
\partial_{t} P_{1}+\partial_{x}\left(\frac{P_{1}^{2}-\left(1+D_{1}^{2}+B_{1}^{2}\right)}{h}\right)=0 \\
\partial_{t} D_{2}+\partial_{x}\left(\frac{B_{3}+D_{2} P_{1}-D_{1} P_{2}}{h}\right)=0 \\
\partial_{t} D_{3}+\partial_{x}\left(\frac{-B_{2}+D_{3} P_{1}-D_{1} P_{3}}{h}\right)=0 \\
\partial_{t} B_{2}+\partial_{x}\left(\frac{-D_{3}+B_{2} P_{1}-B_{1} P_{2}}{h}\right)=0 \\
\partial_{t} B_{3}+\partial_{x}\left(\frac{D_{2}+B_{3} P_{1}-B_{1} P_{3}}{h}\right)=0 \\
\partial_{t} P_{2}+\partial_{x}\left(\frac{P_{1} P_{2}-D_{1} D_{2}-B_{1} B_{2}}{h}\right)=0 \\
\partial_{t} P_{3}+\partial_{x}\left(\frac{P_{1} P_{3}-D_{1} D_{3}-B_{1} B_{3}}{h}\right)=0
\end{array}\right.
$$

respectively. Here we have used the notations

$$
B=\left(B_{1}, B_{2}, B_{3}\right)^{t}, \quad D=\left(D_{1}, D_{2}, D_{3}\right)^{t}, \quad P=\left(P_{1}, P_{2}, P_{3}\right)^{t} .
$$

6.2. Entropy solutions of the ABI system. Obviously, the ABI system is decoupled since the first two equations in (6.8) are nothing but the equations for Chaplygin gas (5.5). Therefore, we may first solve these equations to get $\left(h, P_{1}\right)$, and then determine the remainder unknowns by a linear system of six equations. This is possible for smooth solutions. For weak solutions, we will not do so since the linear system for unknown $\left(D_{2}, D_{3}, B_{2}, B_{3}, P_{2}, P_{3}\right)$ contains discontinuous coefficients which makes the problem difficult to be treated.

Now let us rewrite the system (6.8) by introducing

$$
P_{1}=h v_{2}, D_{2}=h v_{3}, D_{3}=h v_{4}, B_{2}=h v_{5}, B_{3}=h v_{6}, P_{2}=h v_{7}, P_{3}=h v_{8} .
$$

Then the ABI system (6.8) becomes:

$$
\left\{\begin{array}{l}
\partial_{t} h+\partial_{x}\left(h v_{2}\right)=0 \\
\partial_{t}\left(h v_{2}\right)+\partial_{x}\left(h v_{2}^{2}-a^{2} h^{-1}\right)=0 \\
\partial_{t}\left(h v_{3}\right)+\partial_{x}\left(h v_{2} v_{3}-D_{1} v_{7}+v_{6}\right)=0 \\
\partial_{t}\left(h v_{4}\right)+\partial_{x}\left(h v_{2} v_{4}-D_{1} v_{8}-v_{5}\right)=0 \\
\partial_{t}\left(h v_{5}\right)+\partial_{x}\left(h v_{2} v_{5}-B_{1} v_{7}-v_{4}\right)=0 \\
\partial_{t}\left(h v_{6}\right)+\partial_{x}\left(h v_{2} v_{6}-B_{1} v_{8}+v_{3}\right)=0 \\
\partial_{t}\left(h v_{7}\right)+\partial_{x}\left(h v_{2} v_{7}-D_{1} v_{3}-B_{1} v_{5}\right)=0 \\
\partial_{t}\left(h v_{8}\right)+\partial_{x}\left(h v_{2} v_{8}-D_{1} v_{4}-B_{1} v_{6}\right)=0
\end{array}\right.
$$

where $a=\sqrt{1+B_{1}^{2}+D_{1}^{2}}>0$ is a constant. We associate to the system (6.10) the following initial conditions :

$$
t=0: \quad h=h^{0}(x), \quad v_{i}=v_{i}^{0}(x) \quad(2 \leq i \leq 8), \quad x \in \mathbb{R} .
$$


In view of (4.4), we see that (6.10) is a linear Lagrangian system with $n=8$ and $u_{1}=h$. Moreover, let $(t, x) \longmapsto(s, y)=(t, Y(t, x))$ be the change of variables given by

$$
d y=h d x-h v_{2} d t, \quad Y(0, x)=Y_{0}(x) \stackrel{\text { def }}{=} \int_{0}^{x} h^{0}(\xi) d \xi .
$$

Then in Lagrangian coordinates $(s, y)$ the system $(6.10)$ is written as

$$
\partial_{s} \tilde{v}+\tilde{A} \partial_{y} \tilde{v}=0
$$

where $v_{1}=1 / h, v=\left(v_{1}, v_{2}, \cdots, v_{8}\right)^{t}$ and

$$
\tilde{A}=\left(\begin{array}{cccccccc}
0 & -1 & 0 & 0 & 0 & 0 & 0 & 0 \\
-a^{2} & 0 & 0 & 0 & 0 & 0 & 0 & 0 \\
0 & 0 & 0 & 0 & 0 & 1 & -D_{1} & 0 \\
0 & 0 & 0 & 0 & -1 & 0 & 0 & -D_{1} \\
0 & 0 & 0 & -1 & 0 & 0 & -B_{1} & 0 \\
0 & 0 & 1 & 0 & 0 & 0 & 0 & -B_{1} \\
0 & 0 & -D_{1} & 0 & -B_{1} & 0 & 0 & 0 \\
0 & 0 & 0 & -D_{1} & 0 & -B_{1} & 0 & 0
\end{array}\right) .
$$

Notice that $\tilde{A}$ is composed of two block matrix $\tilde{A}=\operatorname{diag}\left(\tilde{A}_{1}, \tilde{A}_{2}\right)$, with $\tilde{A}_{1} \in M_{2}(\mathbb{R})$ being obviously diagonalizable and $\tilde{A}_{2} \in M_{6}(\mathbb{R})$ being symmetric. Hence, $\tilde{A}$ is diagonalizable and the linear system (6.13) is hyperbolic. Furthermore, a straightforward computation gives the eigenvalues of $\tilde{A}$ :

$$
\tilde{\lambda}_{1}=\tilde{\lambda}_{2}=\tilde{\lambda}_{3}=-a, \quad \tilde{\lambda}_{4}=\tilde{\lambda}_{5}=0, \quad \tilde{\lambda}_{6}=\tilde{\lambda}_{7}=\tilde{\lambda}_{8}=a .
$$

Thus applying Proposition 4.1, we obtain

Proposition 6.1. For $h>0$ and any given $B_{1}, D_{1} \in \mathbb{R}$, the $A B I$ system (6.10) is a linear Lagrangian one and hyperbolic. Let $u=h\left(1, v_{2}, \cdots, v_{8}\right)^{t}$. The eigenvalues $\lambda_{i}=\lambda_{i}(u)(1 \leq$ $i \leq 8)$ of the system (6.10) are :

$$
\lambda_{1}=\lambda_{2}=\lambda_{3}=\frac{u_{2}-a}{h}, \quad \lambda_{4}=\lambda_{5}=v_{2}, \quad \lambda_{6}=\lambda_{7}=\lambda_{8}=\frac{u_{2}+a}{h} .
$$

It admits a complete set of classical Riemann invariants $w_{j}=\tilde{l}_{j} v(1 \leq j \leq 8)$, where $\tilde{l}_{j}$ is the left eigenvector of $\tilde{A}$ associated to the eigenvalue $\tilde{\lambda}_{j}$. Moreover, each entropy-entropy flux pair $(E, F)$ of the system is expressed as

$$
(E(u), F(u))=h \sum_{j=1}^{8}\left(1, \lambda_{j}(u)\right) g_{j}\left(\frac{1}{h} \tilde{l}_{j}\left(1, u_{2}, \cdots, u_{8}\right)^{t}\right),
$$

with arbitrary continuous functions $g_{j}(1 \leq j \leq 8)$, and a strictly convex entropy $E(u)$ is given when $g_{j}(\xi)=\xi^{2}$ for all $j=1,2, \cdots, 8$.

Each eigenvalue of the system (6.10) being multiple, then its linear degeneracy can alos be obtained from the result in [1]. To solve the Cauchy problem (6.10)-(6.11), we translate the conditions in (5.7) into

$$
h^{0}, v_{i}^{0} \in L^{\infty}(\mathbb{R})(2 \leq i \leq 8), \quad \inf _{x \in \mathbb{R}}\left(v_{2}^{0}(x)+\frac{a}{h^{0}(x)}\right)>\sup _{x \in \mathbb{R}}\left(v_{2}^{0}(x)-\frac{a}{h^{0}(x)}\right),
$$


which imply that $h(t, x)>0$ for almost all $(t, x) \in \mathbb{R}^{+} \times \mathbb{R}$. As above, for $t \geq 0$, define

$$
X_{0}=Y_{0}^{-1}, \quad Y(t, \cdot)=X^{-1}(t, \cdot),
$$

with

$$
X(t, y)=\frac{1}{2} \int_{0}^{y+t}\left(v_{2}^{0}+\frac{a}{h^{0}}\right)\left(X_{0}(\xi)\right) d \xi-\frac{1}{2} \int_{0}^{y-t}\left(v_{2}^{0}-\frac{a}{h^{0}}\right)\left(X_{0}(\xi)\right) d \xi .
$$

Then applying Theorems 4.1-4.2, we obtain the following result.

Theorem 6.1. Under the condition (6.18), the Cauchy problem (6.10)-(6.11) admits a unique solution $h, v \in L^{\infty}\left(\mathbb{R}^{+} \times \mathbb{R}\right)$ satisfying the entropy equality $\partial_{t} E(u)+\partial_{x} F(u)=0$ in the sense of distributions for all entropy-entropy flux pairs defined by (6.17). This solution is weakly stable in $L^{\infty}\left(\mathbb{R}^{+} \times \mathbb{R}\right)$ and is given by

$$
v_{j}(t, x)=\sum_{i=1}^{8}\left(\tilde{l}_{i} v^{0}\right)\left(X_{0}\left(Y(t, x)-\tilde{\lambda}_{i} t\right)\right) \tilde{r}_{j i} \quad(1 \leq j \leq 8),
$$

where $\tilde{r}_{i}$ is the right eigenvector of $\tilde{A}$ associated to $\tilde{\lambda}_{i}$ such that $\tilde{l}_{i} \tilde{r}_{j}=\delta_{i j}(1 \leq i, j \leq 8)$ and $\tilde{r}_{j i}$ is the $j$-th component of $\tilde{r}_{i}$.

6.3. Entropy solutions of the BI system. It is clear that $(E(u), F(u))$ defined in (6.17) is an entropy-entropy flux pair of the BI system (6.7) when $h$ and $v$ lie in the 4-dimensional sub-manifold (6.2) of $\mathbb{R}^{8}$ (BI manifold following Brenier [9]). By a further computation, we obtain the following result.

Proposition 6.2. For $h>0$ and any given $B_{1}, D_{1} \in \mathbb{R}$, the BI system (6.7) is hyperbolic and linearly degenerate with the eigenvalues

$$
\mu_{1}(U)=\mu_{2}(U)=\frac{P_{1}-a}{h}, \quad \mu_{3}(U)=\mu_{4}(U)=\frac{P_{1}+a}{h},
$$

where $U=\left(B_{2}, B_{3}, D_{2}, D_{3}\right)^{t}, P_{1}$ and $h$ are given by (6.2). It admits a complete set of classical Riemann invariants $W_{i}=L_{i} U / h(1 \leq i \leq 4)$ where $L_{i}(1 \leq i \leq 4)$ are linearly independent constant vectors given by

$$
\left\{\begin{array}{l}
L_{1}=\left(a, \beta_{1}, 0,-\beta_{3}\right), L_{2}=\left(-\beta_{1}, a, \beta_{3}, 0\right), \\
L_{3}=\left(0,-\beta_{2}, a, \beta_{1}\right), L_{4}=\left(\beta_{2}, 0,-\beta_{1}, a\right),
\end{array}\right.
$$

with $\beta_{1}=B_{1} D_{1}, \beta_{2}=1+B_{1}^{2}$ and $\beta_{3}=1+D_{1}^{2}$. Moreover, each entropy-entropy flux pair has an explicit expression:

$$
(E(U), F(U))=h \sum_{i=1}^{4}\left(1, \mu_{i}(U)\right) g_{i}\left(\frac{1}{h} L_{i} U\right),
$$

where $h$ is defined in (6.2) and $g_{i}(1 \leq i \leq 4)$ are arbitrary continuous functions.

Proof. First, replacing $P_{2}$ and $P_{3}$ from the expression $P=D \times B$ and using the notation (6.9), we obtain

$$
v_{7}=B_{1} v_{4}-D_{1} v_{6}, \quad v_{8}=D_{1} v_{5}-B_{1} v_{3} .
$$

Then in Lagrangian coordinates $(s, y)$ with

$$
s=t, \quad d y=h d x-P_{1} d t, \quad h>0,
$$


the BI system (6.7) becomes

$$
\left\{\begin{array}{l}
\partial_{s} \tilde{v}_{3}-\partial_{y}\left(B_{1} D_{1} \tilde{v}_{4}-\left(1+D_{1}^{2}\right) \tilde{v}_{6}\right)=0, \\
\partial_{s} \tilde{v}_{4}+\partial_{y}\left(B_{1} D_{1} \tilde{v}_{3}-\left(1+D_{1}^{2}\right) \tilde{v}_{5}\right)=0, \\
\partial_{s} \tilde{v}_{5}-\partial_{y}\left(\left(1+B_{1}^{2}\right) \tilde{v}_{4}-B_{1} D_{1} \tilde{v}_{6}\right)=0 \\
\partial_{s} \tilde{v}_{6}+\partial_{y}\left(\left(1+B_{1}^{2}\right) \tilde{v}_{3}-B_{1} D_{1} \tilde{v}_{5}\right)=0 .
\end{array}\right.
$$

From Remark 2.1, the BI system (6.7) is equivalent to the linear system (6.27) via the E-L transformation. Now the eigenvalues of (6.27) are

$$
\tilde{\mu}_{1}=\tilde{\mu}_{2}=-a, \quad \tilde{\mu}_{3}=\tilde{\mu}_{4}=a,
$$

with the corresponding left eigenvectors $L_{1}, L_{2}, L_{3}$ and $L_{4}$ given in (6.23). According to (2.11), the eigenvalues of the BI system (6.7) are given by (6.22). Since the vectors $L_{1}$, $L_{2}, L_{3}$ and $L_{4}$ are linearly independent, the linear system (6.27) is hyperbolic. It follows from Proposition 2.1 and Remark 2.1 that the BI system (6.7) is hyperbolic and linearly degenerate. It admits a complete set of classical Riemann invariants $W_{i}=L_{i} U / h=$ $L_{i}\left(v_{3}, v_{4}, v_{5}, v_{6}\right)^{t}(1 \leq i \leq 4)$. Similarly to the proof of $(6.17)$, we may check that each entropy-entropy flux pair of the BI system has the expression (6.24).

Obviously, all entropy solutions $(B, D)$ of the BI system (6.7) satisfy the ABI system (6.8). Conversely, if $(h, B, D, P)$ is a smooth solution of (6.8), with an initial datum in the BI manifold $(6.2)$, then $(B, D)$ is also a smooth solution of $(6.7)$ (see $[3,41])$. This means that the BI-manifold is invariant for smooth solutions. Here we show that it is still invariant for the entropy solution. It implies that if the initial data lie in the BI manifold (6.2), then $(B, D)$ is an entropy solution of (6.7) if and only if $(h, B, D, P)$ is an entropy solution of (6.8). Note finally that the BI system is not strictly hyperbolic. For strictly hyperbolic systems having all linearly degenerate characteristics, we refer to [14] on the existence of entropy solutions.

Theorem 6.2. Let the condition (6.18) be fulfilled and $(h, B, D, P) \in L^{\infty}\left(\mathbb{R}^{+} \times \mathbb{R}\right)$ be an entropy solution of the ABI system (6.8) with initial data $\left(h^{0}, B^{0}, D^{0}, P^{0}\right)$. If $\left(h^{0}, B^{0}, D^{0}, P^{0}\right)$ lies in the BI manifold (6.2), then $(B, D)$ is an entropy solution of the BI system (6.7).

We first show the following lemmas.

Lemma 6.1. For the ABI system (6.8), under the assumptions of Theorem 6.2, we have

$$
P_{2}=B_{1} D_{3}-D_{1} B_{3}, \quad P_{3}=D_{1} B_{2}-B_{1} D_{2} .
$$

Proof. Let $e_{2}=P_{2}-\left(B_{1} D_{3}-D_{1} B_{3}\right)$ and $e_{3}=P_{3}-\left(D_{1} B_{2}-B_{1} D_{2}\right)$. We want to show $e_{2}=0$ and $e_{3}=0$.

By the definition (6.9), we have

$$
e_{2}=h v_{7}-B_{1} h v_{4}+D_{1} h v_{6} \quad \text { and } \quad e_{3}=h v_{8}-D_{1} h v_{5}+B_{1} h v_{3} .
$$

Therefore, using the system (6.10) we get

$$
\partial_{t} e_{2}+\partial_{x}\left(v_{2} e_{2}\right)=0 \text { and } \partial_{t} e_{3}+\partial_{x}\left(v_{2} e_{3}\right)=0
$$


In the coordinates $(s, y)$ they are equivalent to

$$
\partial_{s}\left(\frac{\tilde{e}_{2}}{\tilde{h}}\right)=0 \quad \text { and } \quad \partial_{s}\left(\frac{\tilde{e}_{3}}{\tilde{h}}\right)=0 .
$$

Since the initial data $\left(h^{0}, B^{0}, D^{0}, P^{0}\right)$ lie in the BI manifold (6.2), we deduce that $e_{2}(0, x)=$ $e_{3}(0, x)=0$. Hence, $\tilde{e}_{2}=\tilde{e}_{3}=0$ and then $e_{2}=e_{3}=0$.

Lemma 6.2. For the ABI system (6.8), let us introduce

$$
\begin{gathered}
E_{1}(u)=\frac{1+|B|^{2}+|D|^{2}+|P|^{2}}{h}, \quad F_{1}(u)=\frac{h P_{1} E_{1}(u)-2 a^{2} P_{1}+2 a^{2} h E_{2}(u)}{h^{2}}, \\
E_{2}(u)=\frac{B_{3} D_{2}-B_{2} D_{3}}{h}, \quad F_{2}(u)=\frac{P_{1} E_{2}(u)}{h}-\frac{P_{1}^{2}+a^{2}-h E_{1}(u)}{2 h^{2}} .
\end{gathered}
$$

If (6.29) holds, then $\left(E_{1}, F_{1}\right)$ and $\left(E_{2}, F_{2}\right)$ are two entropy-entropy flux pairs of the $A B I$ system (6.8).

As the proof of Lemma 6.2 involves only elementary calculations, it is postponed to Appendix.

Proof of Theorem 6.2. Let us denote by

$$
\bar{P}=D \times B, \quad \bar{h}=\sqrt{1+|B|^{2}+|D|^{2}+|P|^{2}} .
$$

Lemma 6.1 shows that $P_{2}=\bar{P}_{2}$ and $P_{3}=\bar{P}_{3}$. Now we prove that $h=\bar{h}$ and $P_{1}=\bar{P}_{1}$, i.e., $E_{1}(u)=h$ and $E_{2}(u)=h^{-1} P_{1}$. On one hand, since the solution of the ABI system satisfies the entropy equality for all entropy-entropy flux pairs, Lemma 6.2 together with the notation $h^{-1} P_{1}=v_{2}$ gives

$$
\left\{\begin{array}{l}
\partial_{t} E_{1}+\partial_{x}\left(v_{2} E_{1}+\frac{2 a^{2}\left(E_{2}-v_{2}\right)}{h}\right)=0, \\
\partial_{t} E_{2}+\partial_{x}\left(v_{2} E_{2}-\frac{1}{2}\left(v_{2}^{2}+\frac{a^{2}}{h^{2}}-\frac{E_{1}}{h}\right)\right)=0 .
\end{array}\right.
$$

On the other hand, from the first two equations in (6.10), we have

$$
\left\{\begin{array}{l}
\partial_{t} h+\partial_{x}\left(h v_{2}\right)=0, \\
\partial_{t} v_{2}+\frac{1}{2} \partial_{x}\left(v_{2}^{2}-\frac{a^{2}}{h^{2}}\right)=0 .
\end{array}\right.
$$

Let $W=E_{1}-h$ and $Z=2\left(E_{2}-v_{2}\right)$. Subtracting (6.32) and (6.33) and using $h^{-1} E_{1}=$ $h^{-1} W+1$, we obtain

$$
\left\{\begin{array}{l}
\partial_{t} W+\partial_{x}\left(v_{2} W+\frac{a^{2} Z}{h}\right)=0, \\
\partial_{t} Z+\partial_{x}\left(v_{2} Z+\frac{W}{h}\right)=0 .
\end{array}\right.
$$

In Lagrangian coordinates $(s, y)$, this system is equivalent to

$$
\left\{\begin{array}{l}
\partial_{s}\left(\frac{\tilde{W}}{\tilde{h}}\right)+a^{2} \partial_{y}\left(\frac{\tilde{Z}}{\tilde{h}}\right)=0, \\
\partial_{s}\left(\frac{\tilde{Z}}{\tilde{h}}\right)+\partial_{y}\left(\frac{\tilde{W}}{\tilde{h}}\right)=0 .
\end{array}\right.
$$


Since $\left(h^{0}, B^{0}, D^{0}, P^{0}\right)$ lies in the BI manifold (6.2) which implies that $\tilde{W}=\tilde{Z}=0$ at $s=0$, we deduce from the uniqueness of solutions to the linear system that $\tilde{W}=\tilde{Z}=0$. Hence, $E_{1}(u)=h$ and $E_{2}(u)=v_{2}$. This shows that $h=\bar{h}$ and $P_{1}=\bar{P}_{1}$. Then $(h, B, D, P)$ lies in the BI manifold (6.2) and $(B, D)$ is a weak solution of $(6.7)$. Besides, since $\left(h, P_{1}\right)$ satisfies the conservation law $\partial_{t} h+\partial_{x} P_{1}=0$, we may use the Lagrangian coordinates $(s, y)$ defined in (6.26). In these coordinates the BI system is equivalent to the linear system (6.27) which yields the entropy equality for all entropy-entropy flux pairs $(\tilde{E}, \tilde{F})$. Therefore, Theorem 2.1 implies that $(B, D)$ satisfies the entropy equality for all entropyentropy flux pairs given in (6.24). Thus, $(B, D)$ is an entropy solution of the BI system (6.7).

Since the entropy solution $(B, D)$ of the BI system (6.7) satisfies the ABI system (6.8), from the uniqueness of the entropy solution of the Cauchy problem for the ABI system, we obtain a result of the uniqueness of the entropy solution to the BI system. Moreover, by solving the equations of Chaplygin gas dynamics and the linear system (6.27), we obtain as above an explicit expression of the entropy solution.

Theorem 6.3. Let $U^{0}=\left(B_{2}^{0}, B_{3}^{0}, D_{2}^{0}, D_{3}^{0}\right)^{t} \in L^{\infty}(\mathbb{R})$ and $\left(h^{0}, P^{0}\right)$ be defined by the $B I$ manifold (6.2) satisfying the second condition in (6.18). Then there exists a unique entropy solution $U=\left(B_{2}, B_{3}, D_{2}, D_{3}\right)^{t} \in L^{\infty}\left(\mathbb{R}^{+} \times \mathbb{R}\right)$ to the system (6.7) with the initial data $U^{0}$. The solution is weakly stable in $L^{\infty}\left(\mathbb{R}^{+} \times \mathbb{R}\right)$ and is given by the expressions :

$$
\begin{gathered}
\frac{U(t, x)}{h(t, x)}=\sum_{i=1}^{4}\left(\frac{L_{i} U^{0}}{h^{0}}\right)\left(X_{0}\left(Y(t, x)-\tilde{\mu}_{i} t\right)\right) R_{i}, \\
h(t, x)=\frac{2 a}{w^{0}\left(X_{0}(Y(t, x)+a t)\right)-z^{0}\left(X_{0}(Y(t, x)-a t)\right)},
\end{gathered}
$$

where $X_{0}, Y, L_{i}$ and $\tilde{\mu}_{i}$ are defined by (6.19), (6.20), (6.23) and (6.28), respectively,

$$
z^{0}=v_{2}^{0}-\frac{a}{h^{0}}, \quad w^{0}=v_{2}^{0}+\frac{a}{h^{0}}, \quad v_{2}^{0}=\frac{P_{1}^{0}}{h^{0}},
$$

and $R_{i}$ is defined by $L_{i} R_{j}=\delta_{i j}$ for all $i, j=1,2,3,4$.

Remark 6.1. In one space dimension there is an another way to obtain the existence and uniqueness of entropy solutions of the BI system. Indeed, it suffices to enlarge the $B I$ system by adding the equations for $h$ and $P_{1}$ instead of $h$ and $P$. Then we obtain a linear Lagrangian system of 6 equations of independent variables $h, P_{1},\left(B_{2}, B_{3}\right)$ and $\left(D_{2}, D_{3}\right)$. We may use this system (which is smaller than the ABI system) to replace the $A B I$ system. Here the essential thing is that the relations in (6.25) are linear.

Remark 6.2. The Born-Infeld Lagrangian $L(E, B)$ given in (6.1) is well defined if and only if

$$
\Delta(E, B)=1+|B|^{2}-|E|^{2}-<E, B>^{2} \geq 0
$$

with

$$
E=\frac{1}{h}(D+B \times P), \quad P=D \times B .
$$

Using the definition (6.2) on $h$,

$$
<B \times P, B>=0, \quad<B, P>=<B, D \times B>=0
$$


and the formula

$$
|\xi|^{2}|\eta|^{2}=<\xi, \eta>^{2}+|\xi \times \eta|^{2}, \quad \forall \xi, \eta \in \mathbb{R}^{3}
$$

we have

$$
h^{2} \Delta(E, B)=\left(1+|B|^{2}\right)^{2}+2|D \times B|^{2}-2<D, B \times P>.
$$

Since

$$
<D, B \times P>=<P, D \times B>=|D \times B|^{2},
$$

we obtain finally

$$
h^{2} \Delta(E, B)=\left(1+|B|^{2}\right)^{2}>0 .
$$

Thus, the Born-Infeld Lagrangian $L(E, B)$ is well defined for all $B, D \in \mathbb{R}^{3}$ and all $h>0$. In addition, we have

$$
L(E, B)=-\frac{1}{h}\left(1+|B|^{2}\right)
$$

\section{Appendix : Proof of Lemma 6.2}

Step 1 : Equation for $\left(E_{1}(u), F_{1}(u)\right)$.

Let $v_{2}=h^{-1} P_{1}$. Following Brenier [9], $E_{1}$ satisfies

$$
\partial_{t} E_{1}+\partial_{x}\left(v_{2} E_{1}\right)=2 \partial_{x}\left(\frac{P_{1}-h E_{2}+B_{1}<B, P>+D_{1}<D, P>}{h^{2}}\right) .
$$

Using Lemma 6.1, we get

$$
\begin{aligned}
& B_{1}<B, P>+D_{1}<D, P> \\
= & B_{1}\left(B_{1} P_{1}+B_{2} P_{2}+B_{3} P_{3}\right)+D_{1}\left(D_{1} P_{1}+D_{2} P_{2}+D_{3} P_{3}\right) \\
= & \left(B_{1}^{2}+D_{1}^{2}\right) P_{1}+\left(B_{1} B_{2}+D_{1} D_{2}\right)\left(B_{1} D_{3}-B_{3} D_{1}\right)+\left(B_{1} B_{3}+D_{1} D_{3}\right)\left(B_{2} D_{1}-B_{1} D_{2}\right) \\
= & \left(B_{1}^{2}+D_{1}^{2}\right)\left(P_{1}-h E_{2}\right) .
\end{aligned}
$$

Hence,

$$
\partial_{t} E_{1}+\partial_{x}\left(v_{2} E_{1}\right)=2 a^{2} \partial_{x}\left(\frac{P_{1}-h E_{2}}{h^{2}}\right)
$$

namely,

$$
\partial_{t} E_{1}(u)+\partial_{x} F_{1}(u)=0 .
$$

$\underline{\text { Step } 2}$ : Derivation of the equation for $V=h E_{2}$.

From the system (6.8), we obtain

$$
\begin{aligned}
\partial_{t} V= & \partial_{t}\left(B_{3} D_{2}-B_{2} D_{3}\right) \\
= & B_{3} \partial_{t} D_{2}+D_{2} \partial_{t} B_{3}-B_{2} \partial_{t} D_{3}-D_{3} \partial_{t} B_{2} \\
= & -B_{3} \partial_{x}\left(\frac{B_{3}+D_{2} P_{1}-D_{1} P_{2}}{h}\right)-D_{2} \partial_{x}\left(\frac{D_{2}+B_{3} P_{1}-B_{1} P_{3}}{h}\right) \\
& +B_{2} \partial_{x}\left(\frac{-B_{2}+D_{3} P_{1}-D_{1} P_{3}}{h}\right)+D_{3} \partial_{x}\left(\frac{-D_{3}+B_{2} P_{1}-B_{1} P_{2}}{h}\right)
\end{aligned}
$$

and

$$
\partial_{x}\left(v_{2} V\right)=\partial_{x}\left(v_{2}\left(B_{3} D_{2}-B_{2} D_{3}\right)\right) .
$$


Therefore,

$$
\partial_{t} V+\partial_{x}\left(v_{2} V\right)=\varepsilon_{1}+\varepsilon_{2}
$$

with

$$
\begin{aligned}
\varepsilon_{1}=B_{2} \partial_{x}\left(v_{2} D_{3}\right)-B_{3} \partial_{x}\left(v_{2} D_{2}\right)-D_{2} \partial_{x}\left(v_{2} B_{3}\right)+D_{3} \partial_{x}\left(v_{2} B_{2}\right)+\partial_{x}\left(v_{2}\left(B_{3} D_{2}-B_{2} D_{3}\right)\right), \\
\varepsilon_{2}=-B_{2} \partial_{x}\left(\frac{B_{2}}{h}\right)-B_{3} \partial_{x}\left(\frac{B_{3}}{h}\right)-D_{2} \partial_{x}\left(\frac{D_{2}}{h}\right)-D_{3} \partial_{x}\left(\frac{D_{3}}{h}\right) \\
-B_{2} \partial_{x}\left(\frac{D_{1} P_{3}}{h}\right)+B_{3} \partial_{x}\left(\frac{D_{1} P_{2}}{h}\right)+D_{2} \partial_{x}\left(\frac{B_{1} P_{3}}{h}\right)-D_{3} \partial_{x}\left(\frac{B_{1} P_{2}}{h}\right) .
\end{aligned}
$$

By developing $\varepsilon_{1}, \varepsilon_{2}$ and using the definition of $E_{1}$, we get

$$
\varepsilon_{1}=-V \partial_{x} v_{2}
$$

and

$$
\begin{aligned}
\varepsilon_{2} & =\frac{1}{2 h} \partial_{x}\left(B_{2}^{2}+B_{3}^{2}+D_{2}^{2}+D_{3}^{2}+P_{2}^{2}+P_{3}^{2}\right)-\partial_{x}\left[\frac{1}{h}\left(B_{2}^{2}+B_{3}^{2}+D_{2}^{2}+D_{3}^{2}+P_{2}^{2}+P_{3}^{2}\right)\right] \\
& =\frac{1}{2 h} \partial_{x}\left(h E_{1}-P_{1}^{2}\right)+\partial_{x}\left(\frac{a^{2}+P_{1}^{2}}{h}-E_{1}\right) .
\end{aligned}
$$

Hence,

$$
\partial_{t} V+\partial_{x}\left(v_{2} V-\frac{a^{2}}{h}\right)=\left(P_{1}-V\right) \partial_{x} v_{2}+\frac{1}{2 h} \partial_{x}\left(h E_{1}\right)-\partial_{x} E_{1}
$$

Step 3 : Equation for $\left(E_{2}(u), F_{2}(u)\right)$.

By the definition of $V$, we obtain

$$
\partial_{t}\left(h E_{2}\right)+\partial_{x}\left(h v_{2} E_{2}-\frac{a^{2}}{h}\right)-h\left(v_{2}-E_{2}\right) \partial_{x} v_{2}=\frac{1}{2 h} \partial_{x}\left(h E_{1}\right)-\partial_{x} E_{1}
$$

Using the first equation in (6.10) and after simplification, we have

$$
\partial_{t} E_{2}+\partial_{x}\left(v_{2} E_{2}\right)-\frac{a^{2}}{h} \partial_{x}\left(\frac{1}{h}\right)-v_{2} \partial_{x} v_{2}=\frac{1}{2 h^{2}} \partial_{x}\left(h E_{1}\right)-\frac{1}{h} \partial_{x} E_{1} .
$$

Since

we obtain finally

$$
\frac{1}{2 h^{2}} \partial_{x}\left(h E_{1}\right)-\frac{1}{h} \partial_{x} E_{1}=-\frac{1}{2} \partial_{x}\left(h^{-1} E_{1}\right)
$$

$$
\partial_{t} E_{2}(u)+\partial_{x} F_{2}(u)=0 .
$$

This ends the proof of Lemma 6.2.

Acknowledgment The author is very grateful to G.Boillat for informing me timely of references [9, 41], and for helpful discussions on the Born-Infeld theory. He would also like to thank C.Dafermos for reading and revising the manuscript carefully, which considerably improves the presentation of the paper. 


\section{REFERENCES}

[1] G. Boillat, Chocs caractéristiques, C.R. Acad. Sci. Paris, Série A, 274 (1972), 1018-1021.

[2] G. Boillat, Non linear hyperbolic fields and waves, Lecture Notes Math., Vol. 1640, 1-47, SpringerVerlag, 1996.

[3] G. Boillat, Euler's variational equations with independent momentum, Il Nuovo Cimento, 119 B (2004), 839-847.

[4] G. Boillat and T. Ruggeri, Energy momentum, wave velocities and characteristic shocks in Euler's variational equations with application to the Born-Infeld theory, J. Math. Phys. 45 (2004), 3468-3478.

[5] M. Born and L. Infeld, Foundation of the new field theory, Proc. Roy. Soc. London, A144 (1934), 425-451.

[6] F. Bouchut, On zero pressure gas dynamics, Advances in kinetic theory and computing, 171-190, Ser. Adv. Math. Appl. Sci., 22 (1994), World Sci. Publ., River Edge, NJ.

[7] F. Bouchut and F. James, Duality solutions for pressureless gases, monotone scalar conservation laws, and uniqueness, Comm. Partial Differential Equations 24 (1999), 2173-2189.

[8] Y. Brenier and E. Grenier, Sticky particles and scalar conservation laws, SIAM J. Numer. Anal. 35 (1998), 2317-2328.

[9] Y. Brenier, Hydrodynamic structure of the augmented Born-Infeld equations, Arch. Rat. Mech. Anal. 172 (2004), 65-91.

[10] Y. Brenier, A note on deformations of 2D fluid motions using 3D Born-Infeld equations, Monatsh. Math. 142 (2004), 113-122.

[11] Y. Brenier, Order preserving vibrating strings and applications to Electrodynamics and Magnetohydrodynamics, Methods Appl. Anal., 11 (2004), 515-532.

[12] D. Chae and H. Huh, Global existence for small initial data in the Born-Infeld equations, J. Math. Phys. 44 (2003), 6132-6139.

[13] G.Q. Chen, Hyperbolic systems of conservation laws with a symmetry, Comm. Partial Differential Equations 16 (1991), 1461-1487.

[14] G.Q. Chen, The method of quasidecoupling for discontinuous solutions to conservation laws, Arch. Rat. Mech. Anal. 121 (1992), 131-185.

[15] R. Courant and K.O. Friedrichs, Supersonic Flow and Shock Waves, Interscience publishers, NewYork, 1948.

[16] C. Dafermos, Equivalence of referential and spatial field equations in continuum physics, Notes Numer. Fluid Mech., 43 (1993), 179-183.

[17] C. Dafermos, Hyperbolic Conservation Laws in Continuum Physics, Grundlehren der Mathematischen Wissenschaften, 325, Springer-Verlag, 2000.

[18] S. Demoulini, D.M.A. Stuart and A.E. Tzavaras, A variational approximation scheme for threedimensional elastodynamics with polyconvex energy, Arch. Rat. Mech. Anal., 157 (2001), 325-344.

[19] B. Després, Lagrangian systems of conservation laws, Numer. Math. 89 (2001), 99-134.

[20] B. Després and C. Mazeran, Lagrangian gas dynamics in two dimensions and Lagrangian systems, Arch. Rat. Mech. Anal., 178 (2005), 327-372.

[21] W. E and R.V. Kohn, The initial-value problem for measure-valued solutions of a canonical $2 \times 2$ system with linearly degenerate fields, Comm. Pure Appl. Math. 44 (1991), 981-1000.

[22] W. E, Y.G. Rykov and Y.G. Sinai, Generalized variational principles, global weak solutions and behavior with random initial data for systems of conservation laws arising in adhesion particle dynamics, Comm. Math. Phys. 177 (1996), 349-380.

[23] H. Freistühler, Linear degeneracy and shock waves, Math. Z. 207 (1991), 583-596.

[24] I.M. Gel'fand, Some problems in the theory of quasilinear equations, AMS Translation, Ser.2, No. 29, 1963, 295-381.

[25] G.W. Gibbons, Aspects of Born-Infeld theory and string / M-theory, hep-th / 010659.

[26] G.W. Gibbons and C.A.R. Herdeiro, Born-Infeld theory and stringy causality, Phys. Rev. D63 (2001), 064006 .

[27] J. Glimm, Solutions in the large for nonlinear hyperbolic systems of equations, Comm. Pure Appl. Math., 18 (1965), 695-715.

[28] B. Keyfitz and H. Kranzer, A system of strictly hyperbolic conservation laws arising in elasticity theory, Arch. Rat. Mech. Anal. 72 (1980), 219-241. 
[29] P.D. Lax, Hyperbolic systems of conservation laws II, Comm. Pure Appl. Math. 10 (1957), 537-566.

[30] T.P. Liu, Initial-boundary-value problems for gas dynamics, Arch. Rat. Mech. Anal. 64 (1977), 137168.

[31] T.P. Liu and C.H. Wang, On a nonstrictly hyperbolic system of conservation laws, J. Differential Equations. 57 (1985), 1-14.

[32] G. Lochak, Sur la théorie non linéaire des ondes, C.R. Acad. Sci. Paris, Série III, 250 (1960), 1986-1988.

[33] G. Lochak, Un modèle d'équation non linéaire conservant certaines ondes planes, C.R. Acad. Sci. Paris, Série III, 250 (1960), 2146-2148.

[34] T. Nishida, Global solutions for an initial boundary value problem of a quasilinear hyperbolic system, Proc. Jap. Acad. 44 (1968), 642-646.

[35] T. Nishida and J. Smoller, Solutions in the large for some nonlinear hyperbolic conservation laws, Comm. Pure Appl. Math. 26 (1973), 183-200.

[36] Y.J. Peng, Explicit solutions for $2 \times 2$ linearly degenerate systems, Appl. Math. Letters, 11 (1998), 75-78.

[37] T. Qin, Symmetrizing nonlinear elastodynamic system, J. Elasticity, 50 (1998), 245-252.

[38] D. Serre, Un système hyperbolique non linéaire avec des données oscillantes, C.R. Acad. Sci. Paris, Série I, t. 302, no 3, 1986, 115-118.

[39] D. Serre, Intégrabilité d'une classe de système de lois de conservation, Forum Math. 4 (1992), 607-623.

[40] D. Serre, Systèmes de Lois de Conservation II, Diderot, Paris, 1996.

[41] D. Serre, Hyperbolicity of the nonlinear models of Maxwell's equations, Arch. Rat. Mech. Anal. 172 (2004), 309-331.

[42] B. Sévennec, Géométrie des Systèmes Hyperboliques de Lois de Conservation, Mém. Soc. Math. France, No. 56, 1994.

[43] L. Tartar, Compensated compactness and applications to partial differential equations, Nonlinear analysis and mechanics : Heriot-Watt symposium, Vol. IV, 136-212, Research Notes in Math., 39, Pitman, 1979.

[44] D.H. Wagner, Equivalence of the Euler and Lagrangian equations of gas dynamics for weak solutions, J. Differential Equations. 68 (1987), 118-136.

[45] D.H. Wagner, Conservation laws, coordinate transformations, and differential forms, Hyperbolic problems: theory, numerics, applications (Stony Brook, NY, 1994), 471-477, World Sci. Publishing, River Edge, NJ, 1996.

[46] J. Whitham, Linear and Nonlinear Waves, Wiley, New York, 1974.

[47] Ya.B. Zeldovich, Gravitational instability : an approximate theory for large density perturbations, Astron. \& Astrophys., 5 (1970), 84-89. 